\title{
A QUALITATIVE AND QUANTITATIVE EVALUATION OF AMANTADINE IN THE TREATMENT OF PARKINSON'S DISEASE*
}

\author{
J. E. Walker, J. W. Albers, W. W. Tourtellotte, W. G. Henderson, A. R. Potvin \\ and A. SMIrH
}

Department of Neurology, University of Michigan Medical Center, Ann Arbor, Mich. 48104, U.S.A.

(Received 10 March 1971; in revised form 7 September 1971)

\begin{abstract}
Amantadine hydrochloride (adamantanamine, Symmetrel) has been used for several years as an antiviral agent effective in prophylaxis against Asian influenza $\left(A_{2}\right)[1,2]$. Schwab et al [3] first used amantadine in Parkinson's disease after one patient reported remission of her symptoms while taking the drug to prevent influenza. Schwab and his associates treated 163 Parkinsonian patients with 100-300 mg of amantadine daily. Sixty-six per cent of the group exhibited subjective or objective improvement of akinesia, rigidity, and/or tremor, and sustained benefit was noted in over half of the patients for a period of 3-8 months. Weeth et al [4] treated 39 patients in a similar open trial and found mild to moderate improvement in 23 patients ( 59 per cent). Parkes et al [5] conducted a double-blind crossover trial of amantadine ( $200 \mathrm{mg} /$ day) vs. placebo, each given for a fortnight, involving 37 patients. Thirty-five patients completed the trial, and 26 of them expressed a preference for amantadine, five preferred placebo and four had no preference. The symptoms improved in order of greatest frequency were mobility, tremor, facial expression, speech, general well-being and balance. They also evaluated general history, functional disability, walking history, appearance, tremor, rigidity, limb dexterity, mood, time to walk $25 \mathrm{yd}$, and time to write a set phrase. All scores except walking history and rigidity improved significantly $(p<0.05)$ when amantadine was compared to placebo. The mean time required to walk $25 \mathrm{yd}$ was reduced from 29 to $25 \mathrm{sec}$, and the time to write a set phrase decreased from 40 to $37 \mathrm{sec}$. The only significant difference found between the group giving a clear preference for amantadine and the group that did not was age,
\end{abstract}

\footnotetext{
* This study was supported by the E.I. du Pont de Nemours \& Company, the K.H. Campbell Foundation, Grand Rapids, Michigan and an equipment grant from The University of Michigan Institute of Science and Technology.

Reprint requests and correspondence to Dr. W. W. Tourtellotte, Chief, Neurology Service, Veterans Administration Hospital (Wadsworth), Los Angeles, California 90073, and Professor and ViceChairman, Department of Neurology, UCLA School of Medicine, Los Angeles, California 90024.
} 
with the group preferring amantadine having a mean age of $56 \mathrm{yr}$ and the group preferring placebo or giving no preference having a mean age of 64 yr. Factors that did not influence response to amantadine included severity of disease, stereotactic surgery and concomitant medication.

Fieschi et al [6] conducted a similar double-blind study involving 18 patients. A 22 -item rating scale and three timed performance tests were used to evaluate the effects of placebo and amantadine. They found a more marked placebo effect and also a greater effect on rigidity than had been reported by Parkes and his associates. Hunter et al [7] evaluated 17 patients in a double-blind trial. Four of the patients received placebo throughout the 8 weeks of the trial, four received only amantadine (200 $\mathrm{mg}$ daily), four received placebo the first 4 weeks and amantadine the final 4 weeks, and four received amantadine the first 4 weeks and placebo the final 4 weeks. Weekly assessment of physical signs and functional disabilities revealed small but significant differences in some physical signs and no significant differences in disability scores. All but one of the measures of individual signs were improved, but no individual measure was significantly changed.

Every trial of amantadine to date has found evidence of at least some improvement in patients with Parkinson's disease. Patient acceptance has been excellent and the incidence of significant toxic effects has been low in the $200-300 \mathrm{mg} /$ day range [5]. However, estimates of its efficacy have been inconsistent and there is a paucity of information on the quantitative effect of amantadine on various parameters of neurological function.

Our laboratory has been engaged for several years in the development of a battery of tests for the quantitative evaluation of neurological function. We feel that such tests are well suited for use in evaluating therapy in Parkinson's disease. Not only are these measures recorded on interval or ratio scales and sensitive to relatively slight changes, they also offer the advantage of assessing a wide range of neurological function, including measures of the many components of motor, sensory and psychological performance. It is therefore possible to determine the magnitude of effectiveness of the treatment program and the specific nature of improvement that can be expected. The battery of tests is administered by trained paramedical personnel. They are specifically instructed not to discuss the effect of any medication which the patient might be taking, and do not have access to previous scores. Consequently the data are as objective as can be obtained with this type of testing.

A feature complicating the interpretation of most amantadine trials to date involves the concurrent use of standard anti-Parkinsonian medications. Consequently, we have attempted to establish the efficacy of amantadine alone, since it may have a similar mechanism of action to standard medications. Finally, we felt it important to determine in a comprehensive fashion the effects of amantadine on mental state and activities of daily living, functions upon which the patient primarily determines the preference for a treatment when it is non-toxic.

\section{PATIENTS AND METHODS}

\section{Patients}

Consecutive patients applying for anti-Parkinsonian therapy at The University of Michigan Medical Center were asked to participate in the proposed drug trial. The trial design was described and they were told that they would be asked to reduce their 
present medication to the lowest tolerable level, preferably terminating all other medications for the 12 weeks of the study. Patients were screened to eliminate those having concurrent medical problems, questionable diagnoses, or physical disabilities making it impossible for them to stand or transfer to and from a wheel-chair or to commute to the regular re-evaluation examinations. Patients who had undergone stereotactic surgery also were eliminated. Otherwise, patients were accepted irrespective of sex, age, or duration of illness. In total, three patients were eliminated because of questionable diagnosis, three because of previous stereotactic surgery, four because they were satisfied with their current therapy, and one because he later insisted that he would be unable to tolerate the subsequent evaluation examination. Of the final 42 patients accepted in the study, there were 26 males and 16 females, their ages ranging from 48 to $85 \mathrm{yr}$ (mean $65 \mathrm{yr}$ ). The average duration of their disease was $8 \mathrm{yr}$. Thirty-six of the patients were able to discontinue all other traditional anti-Parkinsonian medications prior to and throughout the study. Of the six patients remaining on anti-Parkinsonian therapy, four remained on a trivial dosage (two patients on Pagitane $^{\mathrm{R}}, 1.25$ mg q.d.; one, on $\operatorname{Artane}^{\mathrm{R}}, 2 \mathrm{mg}$ q.d.; and one, on Parsidol ${ }^{\mathrm{R}}, 50 \mathrm{mg}$ once per week); whereas, two patients required a larger dosage (one patient, Artane ${ }^{\mathbf{R}}$, $6 \mathrm{mg}$ q.d., and the other Cogentin ${ }^{\mathrm{R}}, 6 \mathrm{mg}$ q.d.). Because no significant differences were found when the data were analyzed with and without these patients, they have been included in all the analyses.

\section{Trial design}

Patients were instructed to taper their present medications, discontinuing antihistamines immediately and tapering anticholinergics over a 1 week period. Patients who could not tolerate this change were instructed to choose from their medication one tablet twice daily, establishing a routine for at least 1 week prior to their second visit. On the average, patients called the neurologists (Drs. Walker and Tourtellotte) three times during the 'tapering period' and each time the patient was asked: 'Can you tolerate being just like you are, with no additional medication, for 6-12 weeks; that is, can you tolerate being placed on placebo medication?' Prior to removal of their pretrial medication, the battery of qualitative and quantitative neurological examinations were administered to each patient. Various laboratory tests (serology, hematocrit, white blood cell count and differential, BUN, SGOT, alkaline phosphatase and urinalysis) were obtained.

Four weeks after their initial visit each patient returned, having been successfully off all medication or on a stable regimen. An interval history was obtained and their neurological function re-evaluated. Patients were then randomly divided into two groups with one group given $100 \mathrm{mg}$ amantadinc twice daily and the other group given placebo capsules of similar taste and appearance. Three weeks later, patients returned for re-evaluation. An interval history was obtained and neurological function evaluated. Unused capsules were returned and later counted and identified. The amantadine and placebo groups were then reversed. Three weeks later, patients returned for the final trial evaluation. An interval history was obtained, neurological function evaluated, and unused capsules returned. A summary of the experimental paradigm is shown in Table 1 . It is emphasized that the same 42 patients received both treatments. 
TABLE 1. EXPERIMENTAL PARADIgM

\begin{tabular}{cccccc}
\hline Group & No. of & \multicolumn{4}{c}{ Medication taken during week } \\
patients & 1 & $2-5$ & $6-8$ & $9-11$ \\
\hline 1 & 21 & $\mathrm{~S}$ & $\mathrm{O}$ & $\mathrm{A}$ & $\mathrm{P}$ \\
2 & 21 & $\mathrm{~S}$ & $\mathrm{O}$ & $\mathrm{P}$ & $\mathrm{A}$ \\
\hline
\end{tabular}

$\mathrm{S}=$ Standard anti-Parkinsonian medication being used upon entry to trial.

$\mathrm{O}=\mathrm{Off}$ all medications.

$\mathbf{P}=$ Placebo.

$A=$ Amantadine.

\section{Assessment}

Both qualitative and quantitative measures of the patients' functional capacity were obtained throughout the trial. The clinical evaluations were obtained and the quantitative neurological examination was administered on four separate occasions; while the patients were on their standard medications, while they were off all medications or tapered to their lowest tolerable level, and while they were on placebo and amantadine. The Simulated Activities of Daily Living Examination (SADLE) and the Neuro-Psychological Examination (NPE) described below were not administered during the patients' initial visit while they were on their conventional medications but were administered on all subsequent visits. A brief summary of the assessment measures follows:

\section{(A) Qualitative measures \\ (1) Patients' impression}

Patients were asked to subjectively evaluate any change in condition after both placebo and amantadine treatment. Each patient determined (1) whether the drug had been useful, and (2) whether he was worse, no different, 25 per cent improved, 50 per cent improved, 75 per cent improved, or completely well after treatment compared to his pretreatment condition. Patients were also asked to specify in what ways, if any, their condition had changed.

\section{(2) Neurologists' overall subjective impression}

The 'blinded' neurologists were asked to subjectively evaluate their preference for the two treatments. Such a subjective evaluation included their clinical observations as well as their interpretation of reported side effects, of the patients' subjective impressions, and of qualitative evaluation forms described below.

\section{(3) Qualitative cvaluation}

Two subjective evaluation forms were filled out by the same 'blinded' neurologists throughout the trial. The first evaluation required the neurologists to categorize the individual patient's functional disabilities with respect to walking, dressing, hygiene, eating and feeding, and speech using the disability scales shown in Appendix A [8]. A total functional disability score was obtained by adding the scores for the specific areas. The second evaluation required the neurologists to evaluate relevant physical signs (i.e., tremor, rigidity, cogwheeling, weakness, finger dexterity, succession movements, bradykinesia, foot tapping, associated movements, rising, posture, 
stability and gait) using the traditional classifications of normal, minimal, mild, moderate and severe.

(4) Motion pictures of patients performing a routine series of tasks were recorded at each examination period as a permanent visual record of the patients' motor capabilities. The movie sequence included a record of the patient sitting at rest in a chair; performing a series of rapid succession movements (right and left); performing a series of coordinated movements, including picking up a glass and bringing it to his mouth (right and left); tapping his feet; repeatedly rising and sitting in a chair; standing without support; walking; and turning.

\section{(B) Quantitative measures}

(1) An Activities of Daily Living (ADL) test battery was administered to assess the patients' performance on standardized tests which simulate acts of daily living (SADLE). The time necessary to complete individual tests such as putting on a shirt, tying a bow, using buttons, dialing a telephone, manipulating a safety pin, opening an envelope, opening a door, vocalizing, etc., was recorded. The test names along with their abbreviations and a brief description of each test are shown in Appendix B. These tests were administered by a trained 'blind' occupational therapist (J. A. Sagath) whose only responsibility was to obtain data. She was instructed not to discuss the effect of medication and she had no access to previous scores.

(2) A Clinical Quantitative Neurological Examination (CQNE) was administered to each patient. This battery of motor and sensory tests assesses basic abilities including strength, steadiness, simple reaction time, hand and foot speed, hand and foot coordination, psychomotor coordination, manual and finger dexterity, gait and station, and fatigue. The sensory tests include evaluation of visual acuity, touch sensation, vibration sensation, 2-point discrimination, and position sense. These tests are more completely described in previous articles [9-11], and more recently in Potvin's Ph.D. Thesis (see footnotes in Appendix B and C). A brief description of each test appears in Appendix $\mathrm{C}$, including the measures, the units, and the instrumentation. These tests were administered by a trained 'blind' physical therapist (Kazmicrezak or Shimp) whosc only responsibility was to obtain data. (She was instructed not to discuss the effect of medication and she had no access to previous scores.)

(3) A neuro-psychological test battery was administered to evaluate specific higher and lower level cerebral functions (NPE).

The tests included the Peabody Picture Vocabulary test, a color naming and recognition subtest from the Eisenson test for Aphasia, a double simultaneous (face-hand) stimulation test, a discrimination test of right-left and body parts, the Raven Coloured Progressive Matrices test, the Hooper Visual Organization test, a written and oral digit substitution test, and the Wechsler Adult Intelligence Scale subtests (digit span, digit-symbol, picture completion, picture arrangement, and object assembly). These tests were administered by a trained 'blind' psychologist (A. Smith or R. Champoux) whose only responsibility was to obtain data. He was instructed not to discuss the 
effect of medication and he had no access to previous scores. The results of this portion of the trial will be reported more completely elsewhere.

\section{Control subjects}

Normative data previously were established for all of the quantitative tests except the activities of daily living (SADLE) items using 80 young asymptomatic subjects, mean age $21.5 \mathrm{yr}$, S.D. $-2.2 \mathrm{yr}$. Normative data for the (SADLE) were obtained using 23 spouses of patients participating in the amantadine trial. The mean age of the control group was $61.5 \mathrm{yr}$, S.D. $=3.8 \mathrm{yr}$. Normal scores are included in Tables 4 and 7.

\section{RESULTS}

\section{Patients' impressions}

Patients were asked to evaluate their condition at the end of placebo and amantadine treatment. Responses to the question: 'Was the drug useful to you ?' are summarized in Table 2 for both placebo and amantadine treatment. There was a significant tendency for amantadine treatment to be subjectively classified as useful by the patients as compared to placebo treatment $(p<0.01$ using the McNemar test for correlated proportions), with 64 per cent of the patients responding favorably to amantadine treatment as compared to 21 per cent of the patients responding favorably to placebo treatment.

TABle 2. Results of aDministration of Placebo and amantadine TO 42 PARKINSONIAN PATIENTS. PATIENTS' OVERALl SUBJECTIVE IMPRESSIONS. PATIENTS' ReSPONSE TO: 'WAS THE DRUG USEFUl TO YOU?'

\begin{tabular}{lccc}
\hline \multirow{2}{*}{$\begin{array}{l}\text { Patients' response after } \\
\text { placebo treatment }\end{array}$} & \multicolumn{2}{l}{$\begin{array}{l}\text { Patients' response after } \\
\text { amantadine treatment }\end{array}$} & \\
& Useful & Not useful & Row total \\
\hline Useful & $7 \%(3)^{*}$ & $14 \%(6)$ & $21 \%(9)$ \\
Not useful & $57 \%(24)$ & $21 \%(9)$ & $78 \%(33)$ \\
$\quad$ Column total & $64 \%(27)$ & $35 \%(15)$ & \\
\hline
\end{tabular}

*Indicates $7 \%$ ( 3 patients) found the drug useful while on placebo treatment and useful while on amantadine treatment.

The patients' subjective overall evaluations of change in condition are summarized in Fig. 1, demonstrating that a greater number of patients responded 'worse' or 'no change' following placebo treatment than following amantadine treatment while fewer patients responded they were improved following placebo treatment than following amantadine treatment.

Patient impressions of specific improvements are summarized in Table 3. Examination of this table suggests that administration of amantadine did not result in improvement in any one specific area, although the most frequent patient responses included improved walking, faster movements, less tremor, increased ability to get out of a chair and bed, and clearer speech. Patient impressions after receiving placebo treatment most frequently involved decreased tremor. 


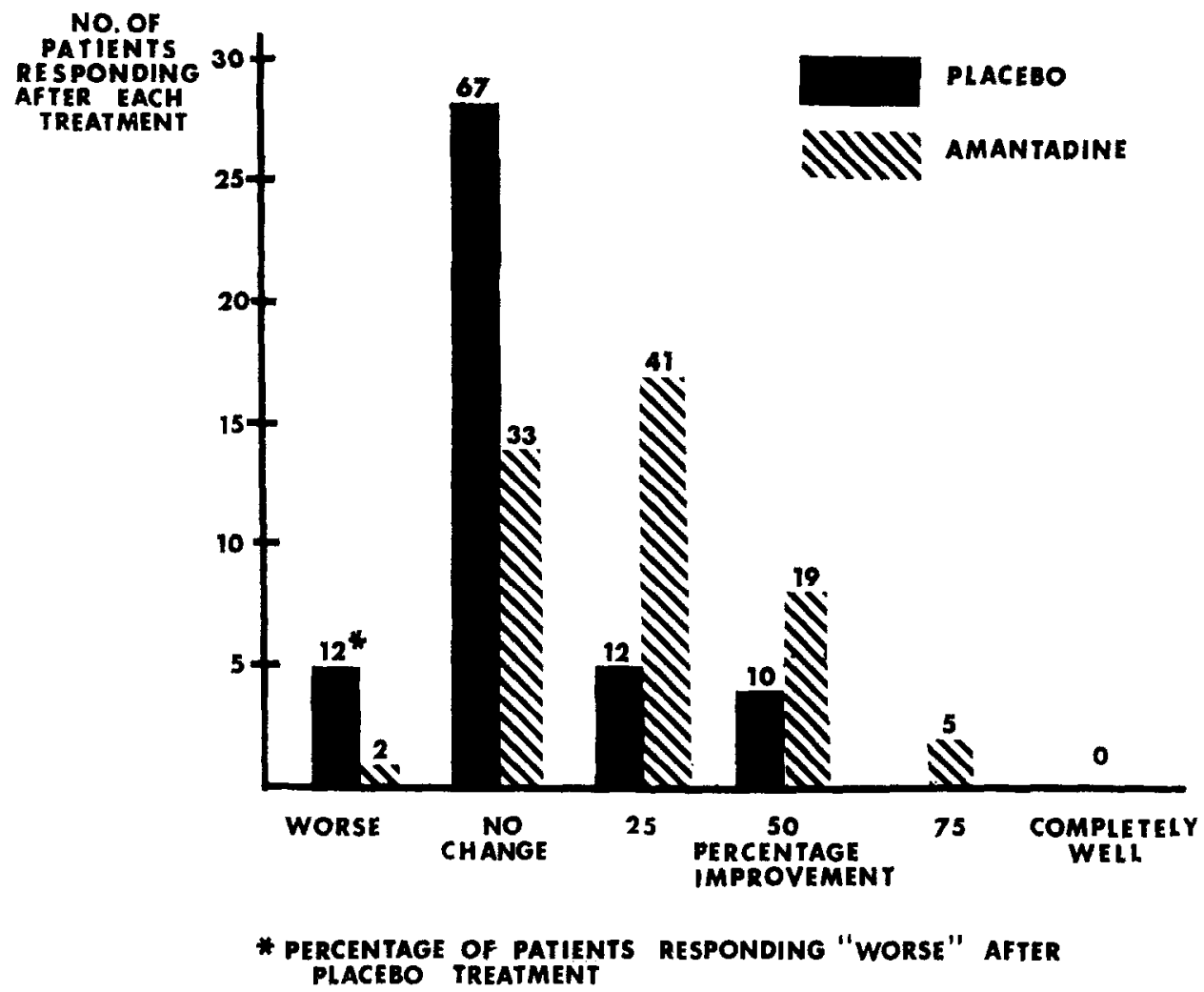

FIG. 1. Patients' overall subjective evaluations. Patients' responses to the question: 'Are you worse, no different, 25 per cent improved, 50 per cent improved, 75 per cent improved, or completely well after treatment as compared to your pretreatment condition?"

\section{Neurologists' overall subjective impressions}

The subjective clinical impression was that amantadine had a moderate but positive effect upon the majority of patients as compared to placebo treatment. Using information recorded during the interval histories, the neurologists were asked to distinguish between placebo and amantadine, responding to the question 'Which is the better drug in the treatment of the individual patient?' Amantadine was preferred over placebo for 74 per cent of the patients ( 31 patients). Utilizing the sign test, this is a significant impression $(p<0.01)$.

\section{Qualitative evaluation sheets}

General Functional Disability Scores are shown in Fig. 2, comparing average scores obtained while the patients were on their previous medication, pretreatment, placebo and amantadine. These qualitative measures of activities of daily living indicate by inspection that amantadine treatment has a modest but positive effect, being better than placebo treatment, pretreatment, or the previous medication in each of the categories evaluated. Utilizing the sign test a significant difference $(p<0.05)$ between amantadine and placebo treatment existed for walking, hygiene, eating and 
Table 3. Patients' subjective evaluation. Patients' responses to: 'HOW ARE YOU SPECIFICALLY IMPROVED?'

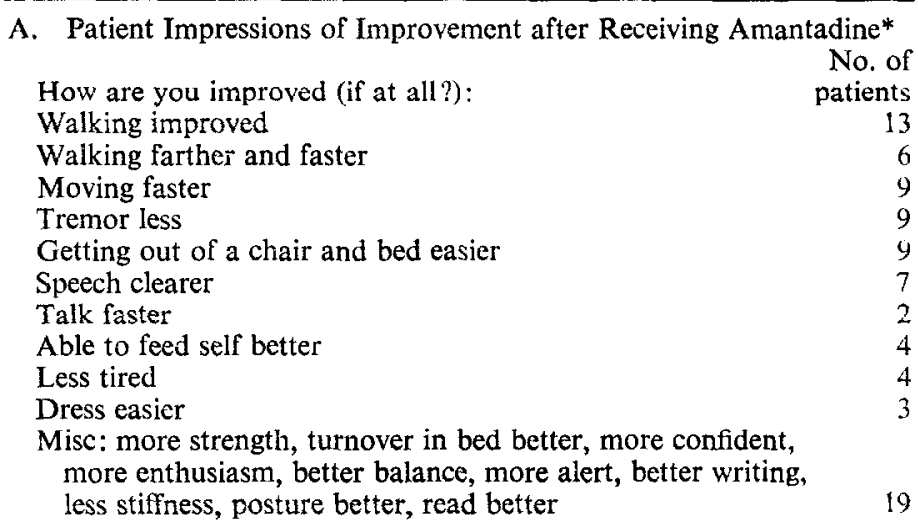

B. Patient Impressions of Improvement after Receiving Placebo

How are you improved (if at all?):

No. of

Tremor less

patients

Walking improved

Mood better

Misc: more strength, less tired, speech clearer, able to do more, balance better, more alert, able to put hand in back pocket, more mobile, feel better generally, less constipated, sleep better 10

\footnotetext{
*Patients asked, 'In what specific ways are you better, if any?' The categories listed are paraphrased reductions of the patients' own comments. Some patients are entered into several specific categories of improvement.
}

feeding, speech and total disability score, but not for dressing. Additional subjective evaluations using the more detailed qualitative evaluation forms are summarized in Fig. 3. Mean scores by inspection indicate that amantadine treatment was preferable over placebo treatment for 17 of the 18 items evaluated. The greatest improvement again was recorded for the evaluation of gait. Significant improvement of amantadine over placebo (sign test, $p<0.05$ ) was found for tremor of the head, succession movements of the upper extremities, weakness of the lower extremities, rising from a chair, posture and gait. Only foot tapping showed more patients worsened on amantadine, but this was not significant.

\section{Quantitative measures}

Simulated activities of daily living eximination (SADLE). Results of the tests of the SADLE are summarized in Table 4. Scores are shown for 23 asymptomatic spouse controls and 42 parkinsonian patients prior to treatment (after the patients had been tapered to minimal or no medication). In addition, scores obtained following administration of placebo and amantadine are shown for both treatment groups separately as well as the combined group. The results of the paired $t$-tests comparing placebo to amantadine treatment for each of the test items also are shown. Fourteen of the 19 items indicate significantly better patient performance after amantadine treatment when compared to placebo treatment for the combined groups. Inspection of the 


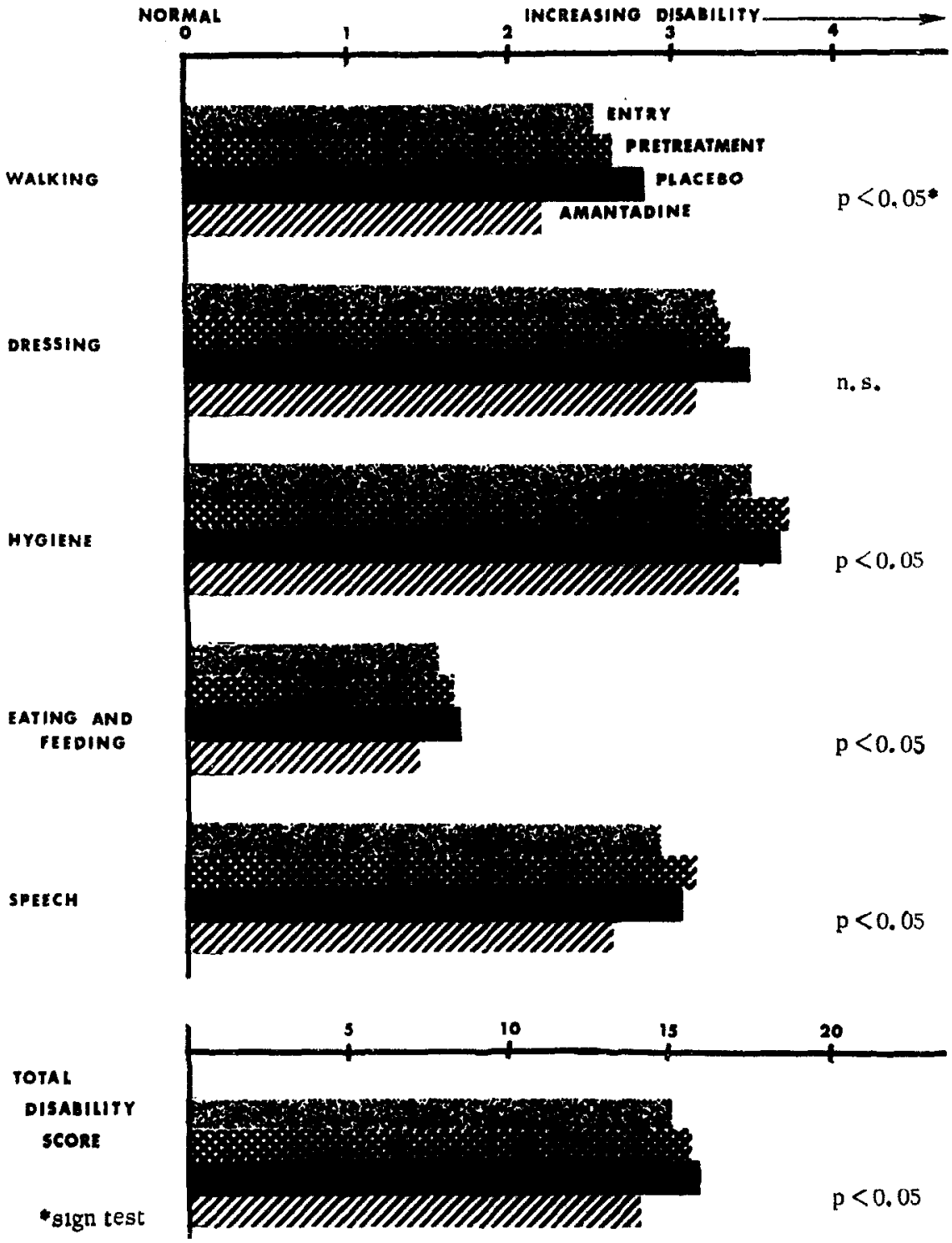

FiG. 2. General functional disability scores comparison of previous medication (entry), pretreatment placebo and amantadine. Neurologists' evaluations by inquiry of activities of daily living, mean scores for all $\mathbf{4 2}$ patients. (See Appendix A for data sheet used.) 


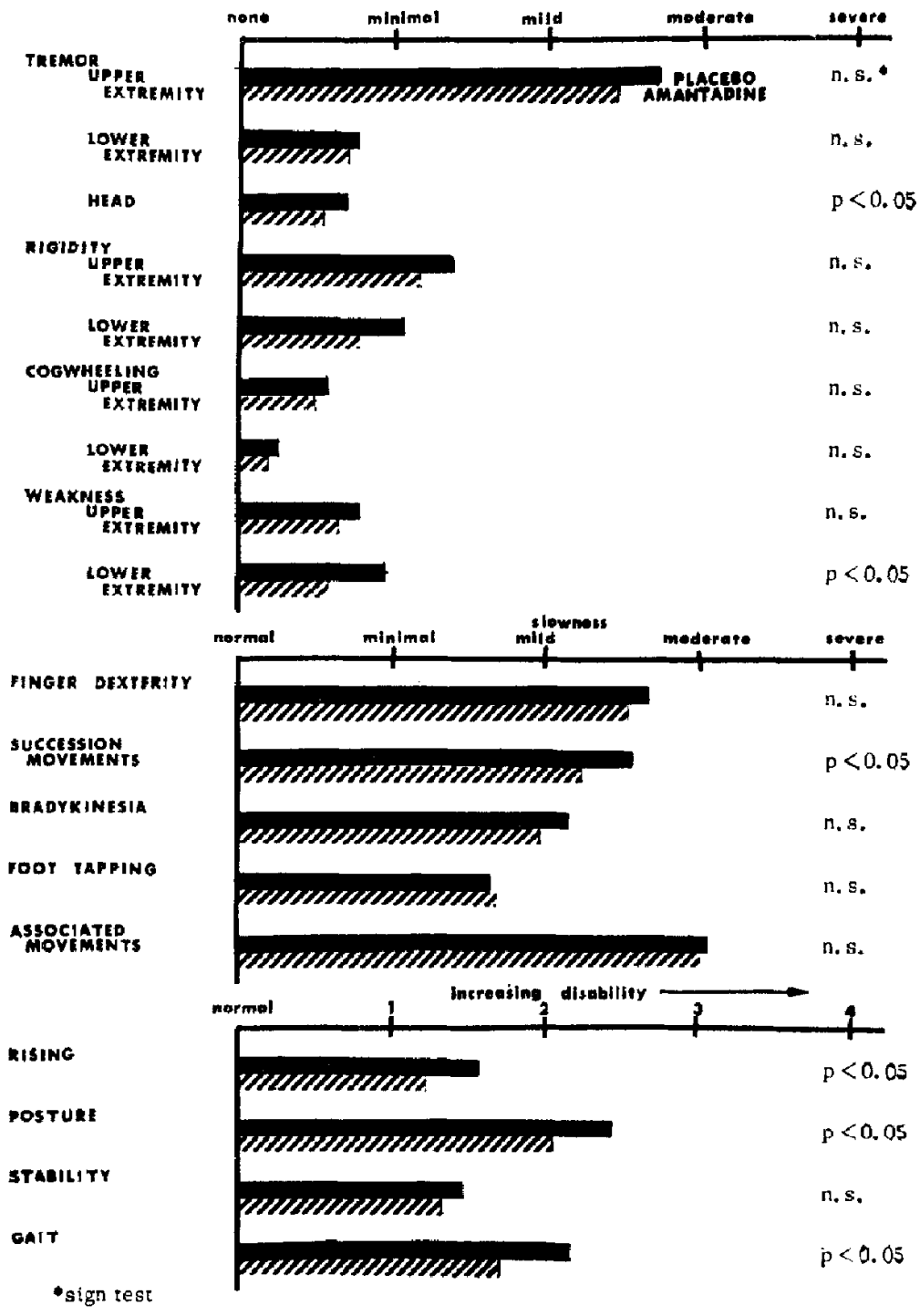

Frg. 3. Evaluation of relevant physical signs of Parkinsonism after placebo and amantadine treatment. Neurologists' subjective evaluation. Mean scores for all 42 patients. 


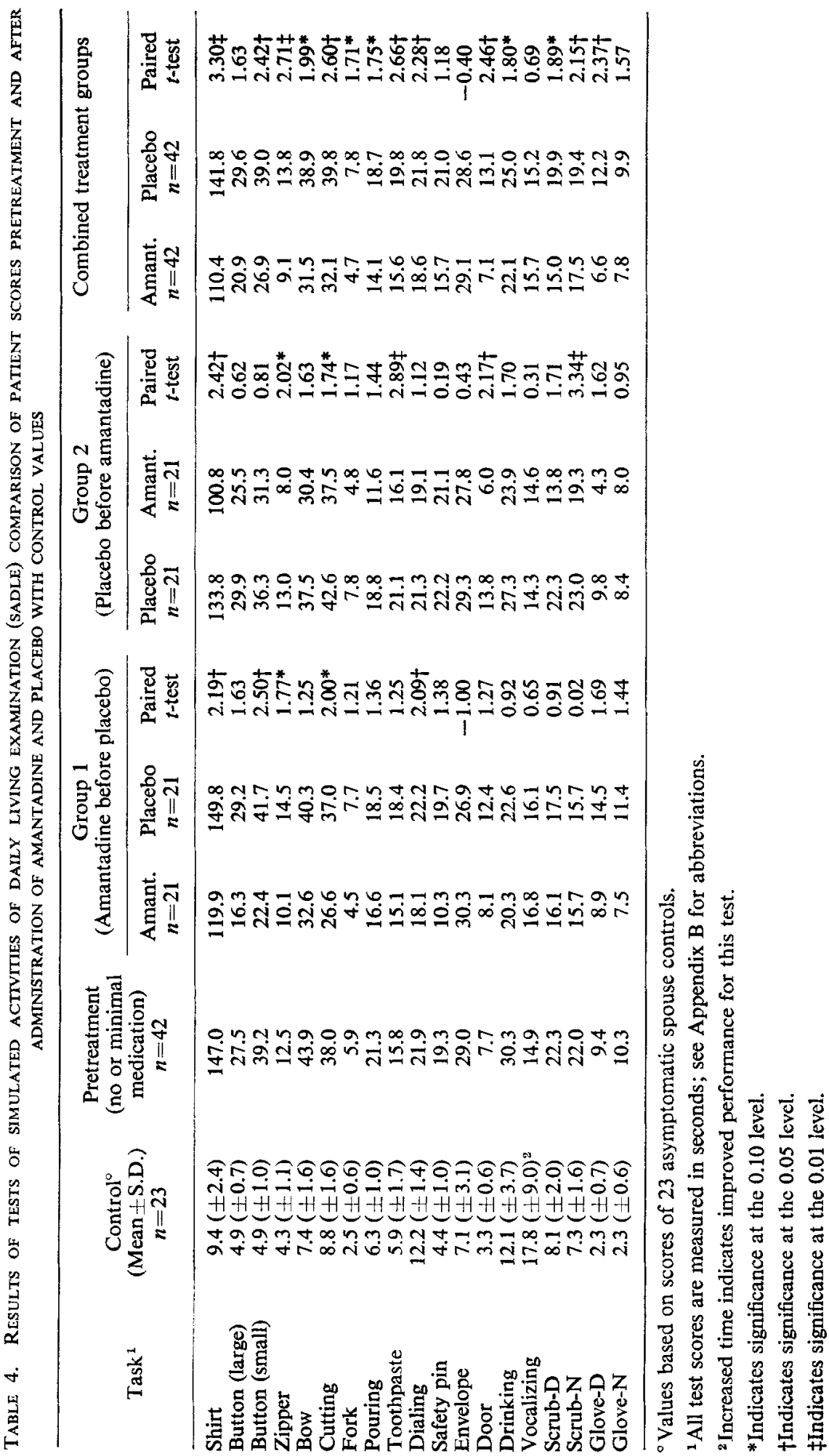


160 J.E. Walker, J.W. Albers, W.W. Tourtellotte, W.G. Henderson, A.R. Potvin and A. Smith

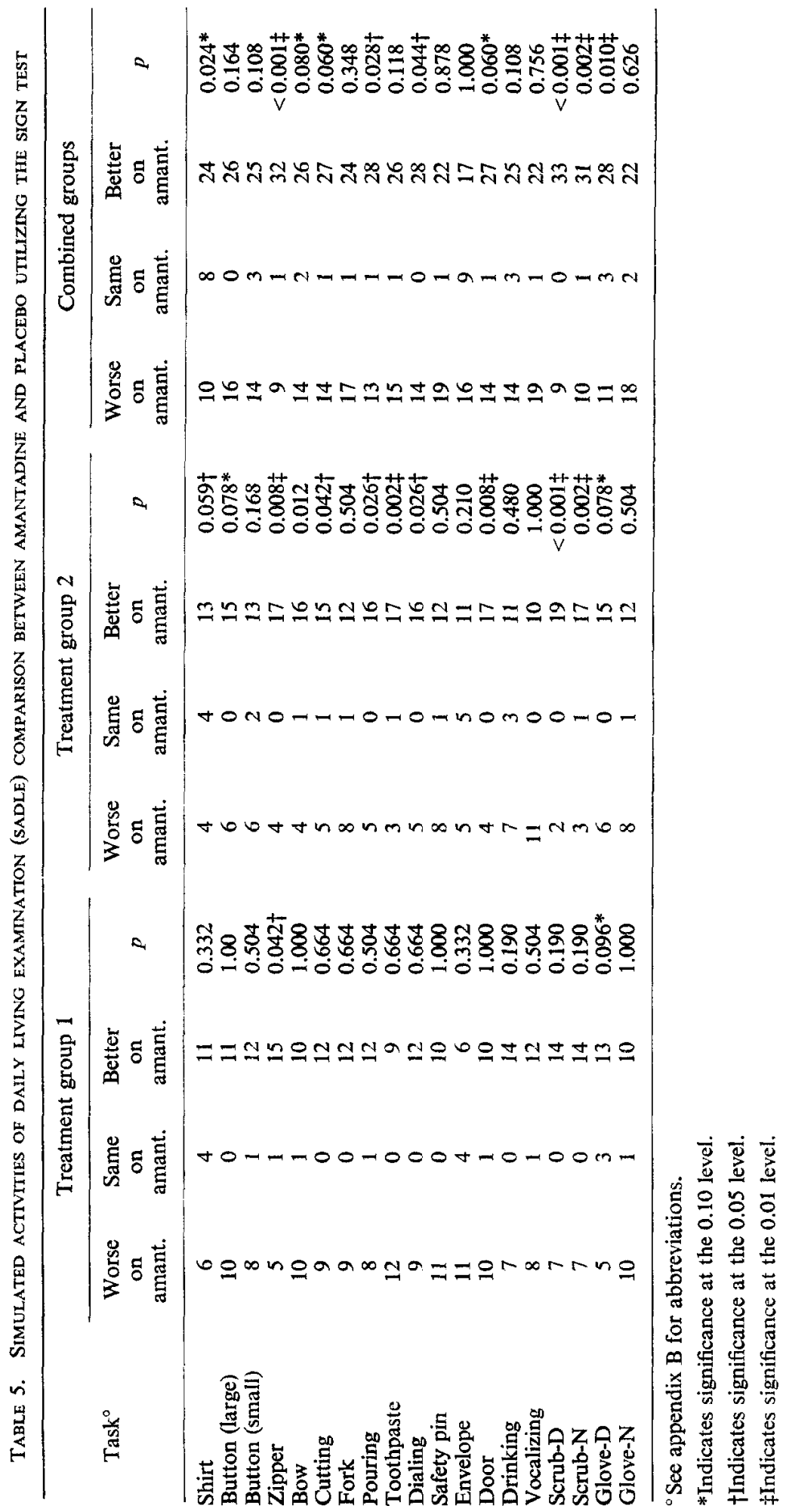


remaining five test items reveals that all but one (envelope) favor amantadine treatment. Examination of the scores for treatment Groups 1 and 2 indicates no striking differences between the two groups.

Table 5 summarizes the results of a sign test analysis, comparing the number of patients better on amantadine treatment to the number better after placebo treatment for the individual and combined treatment groups. Any measurable improvement or deterioration in performance was regarded as a change for the purposes of this analysis. Ten of the 19 test items demonstrate that a significantly greater number of patients performed better after amantadine treatment than after placebo treatment for the combined groups. Inspection of the remaining nine tests demonstrates a similar trend but not at a significant level. Separate comparison of treatment Groups 1 and 2 indicates that Group 2 (treated with placebo before amantadine) demonstrated a more significant preference for amantadine than did Group l (treated with amantadine before placebo). This finding may be interpreted as an indication that learning effects in these tests, although slight, are significant. However, the nature of the crossover trial minimizes this effect when the two treatment groups are combined for analysis.

In order to determine the magnitude of response to placebo and amantadine, the SADLE test scores are better expressed as a percentage of age matchcd normal

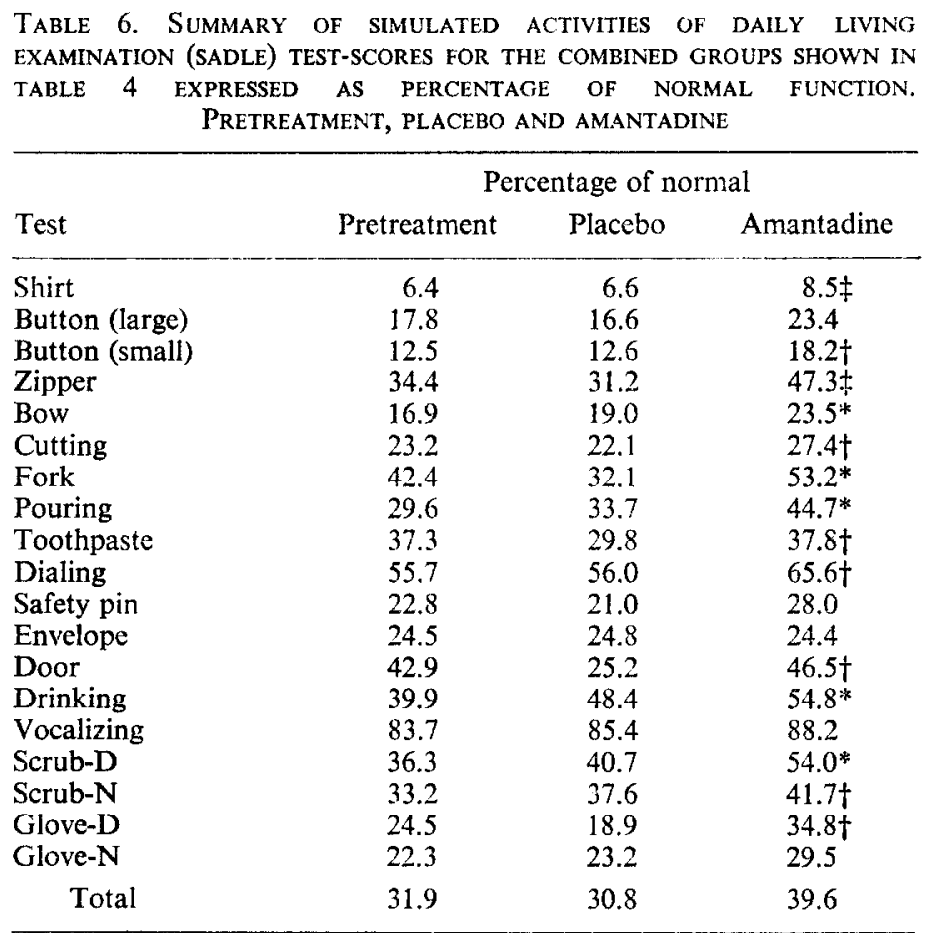

\footnotetext{
*Indicates significance at the 0.10 level, placebo vs. amantadine (paired $t$-test).

IIndicates significance at the 0.05 level, placebo vs. amantadine (paired $t$-test).

$\ddagger$ Indicates significance at the 0.01 level, placebo vs. amantadine (paired $t$-test).
} 


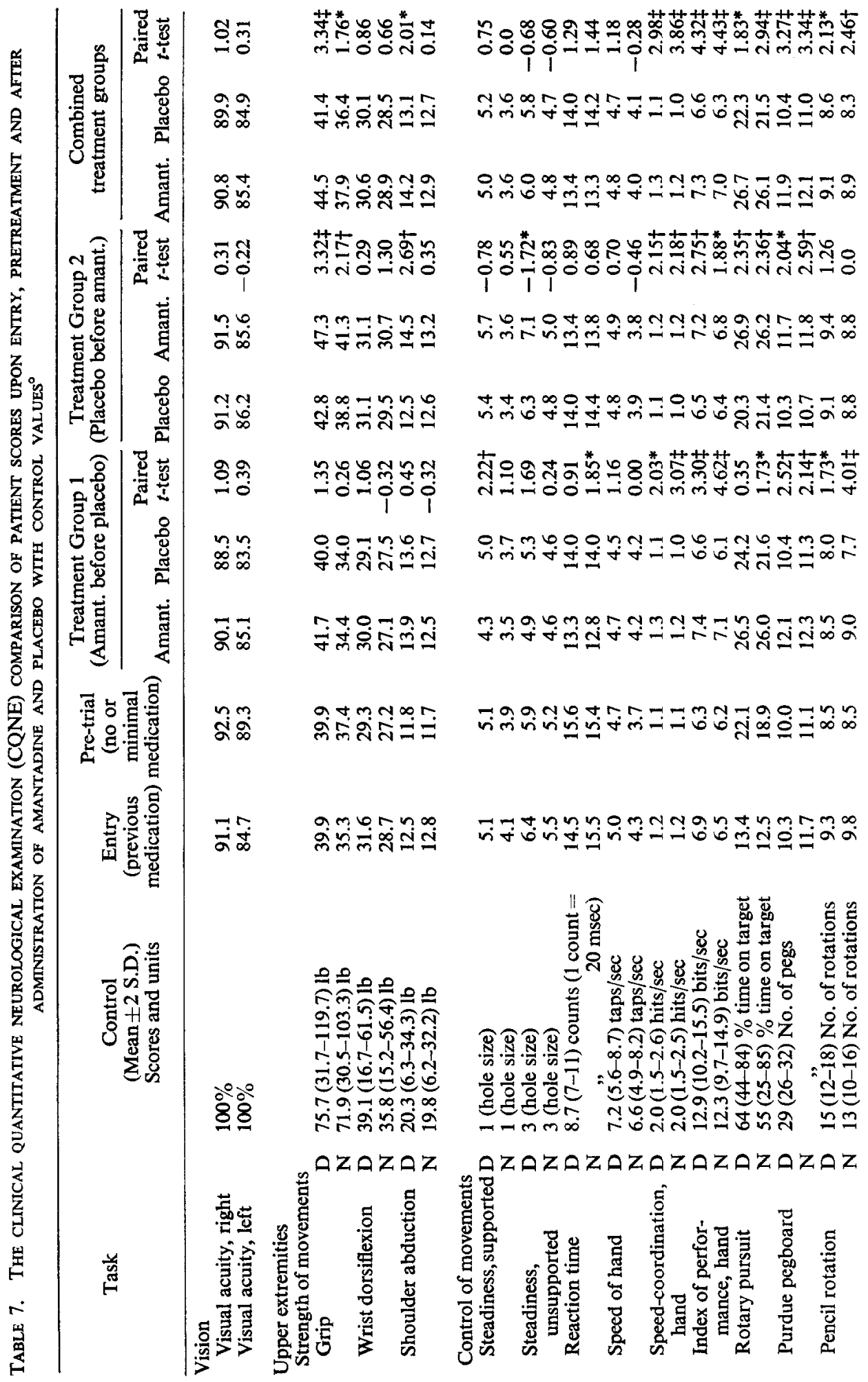




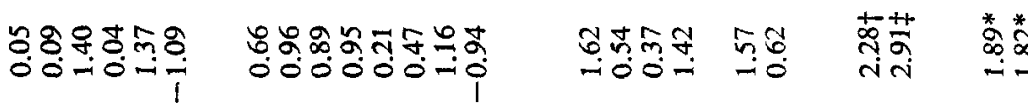

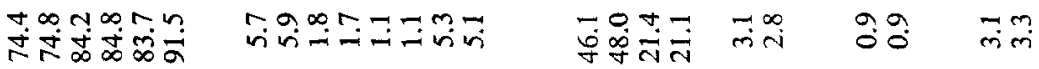

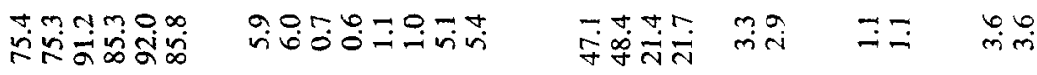

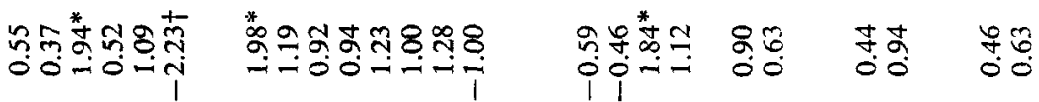
1

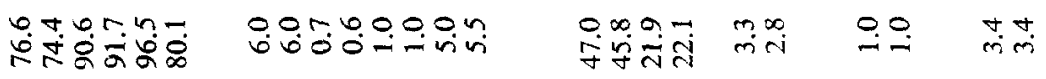

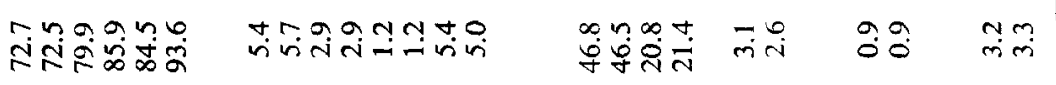

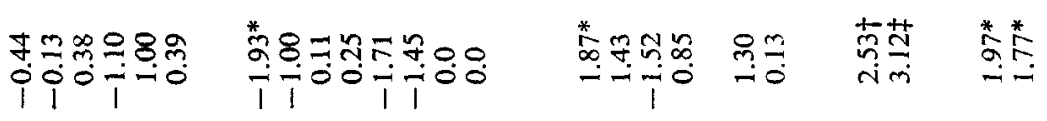

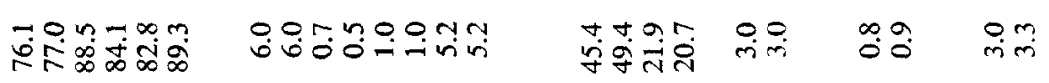

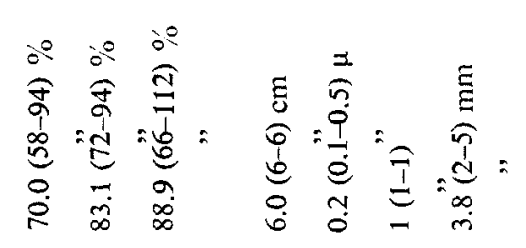




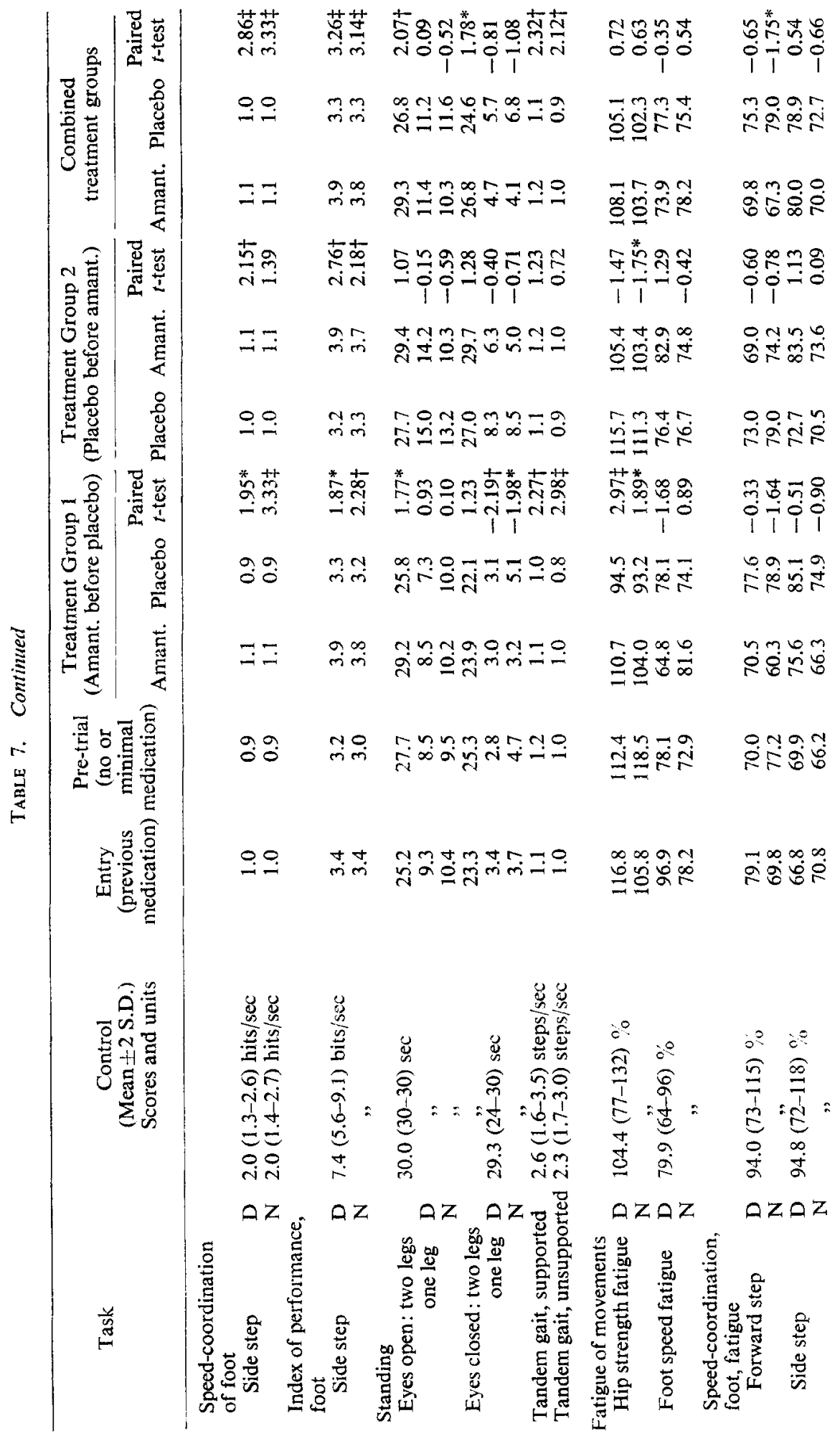




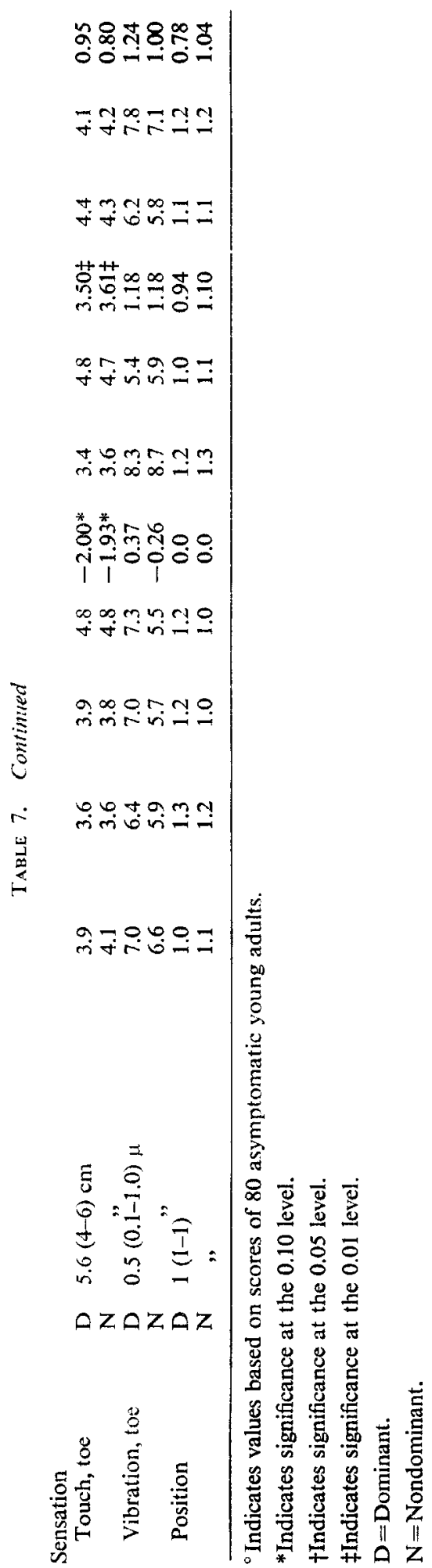




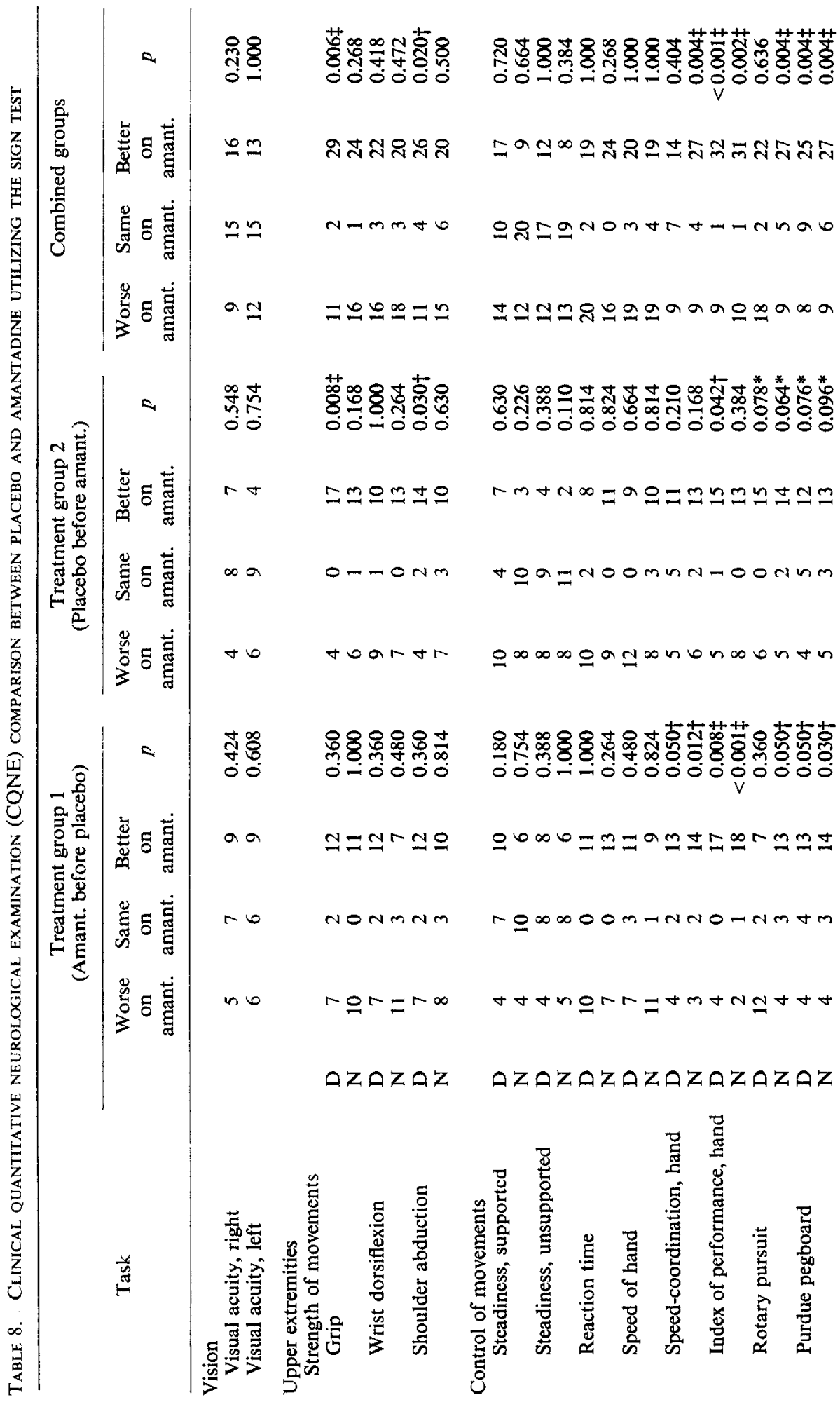


器雚

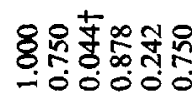

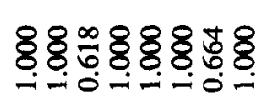

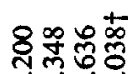

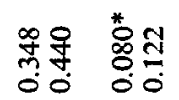

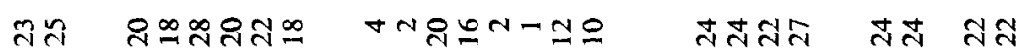

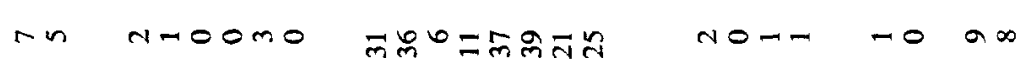

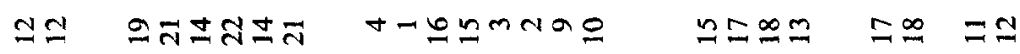

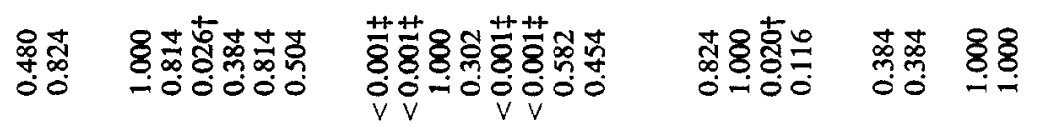

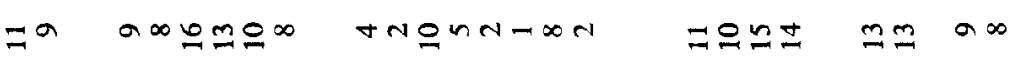

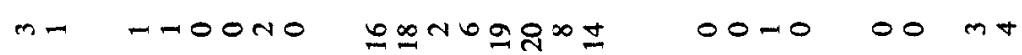

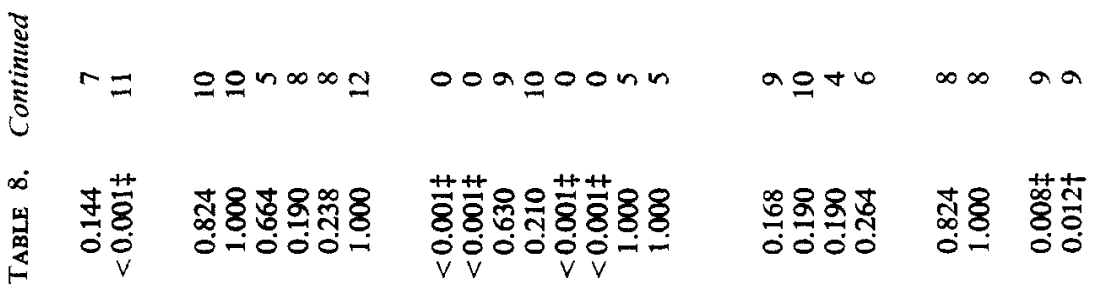

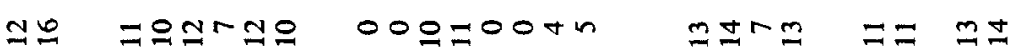

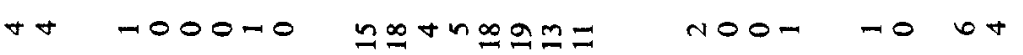

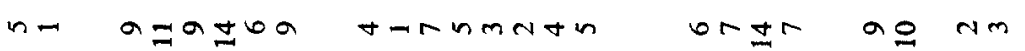
AZ คZคZAZ คZคZคZคZ คZคZ คZ คZ

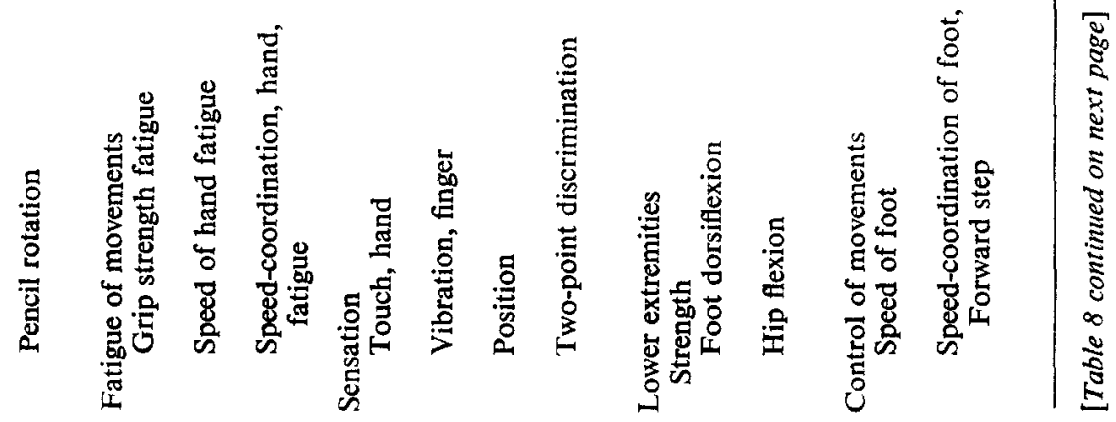




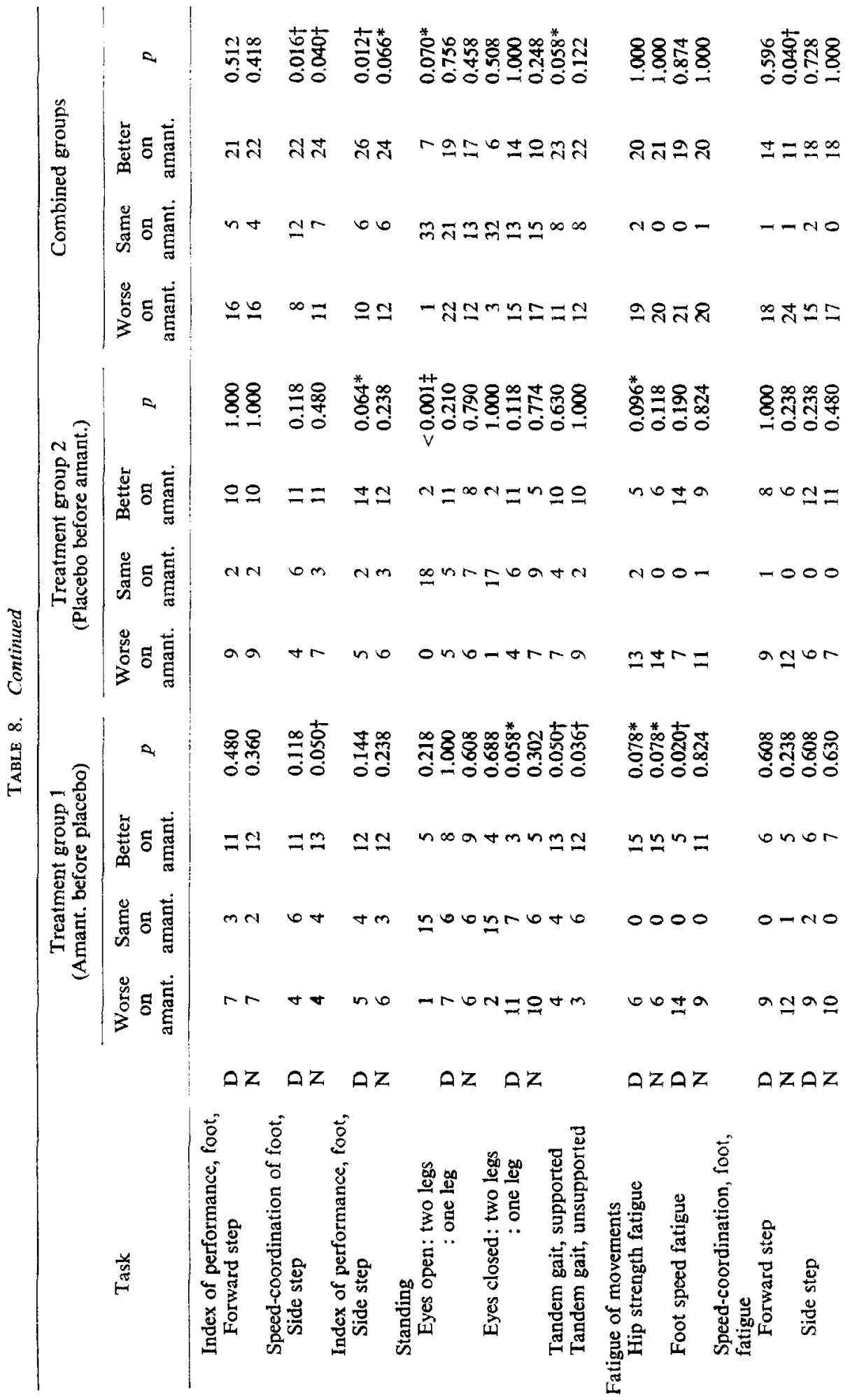




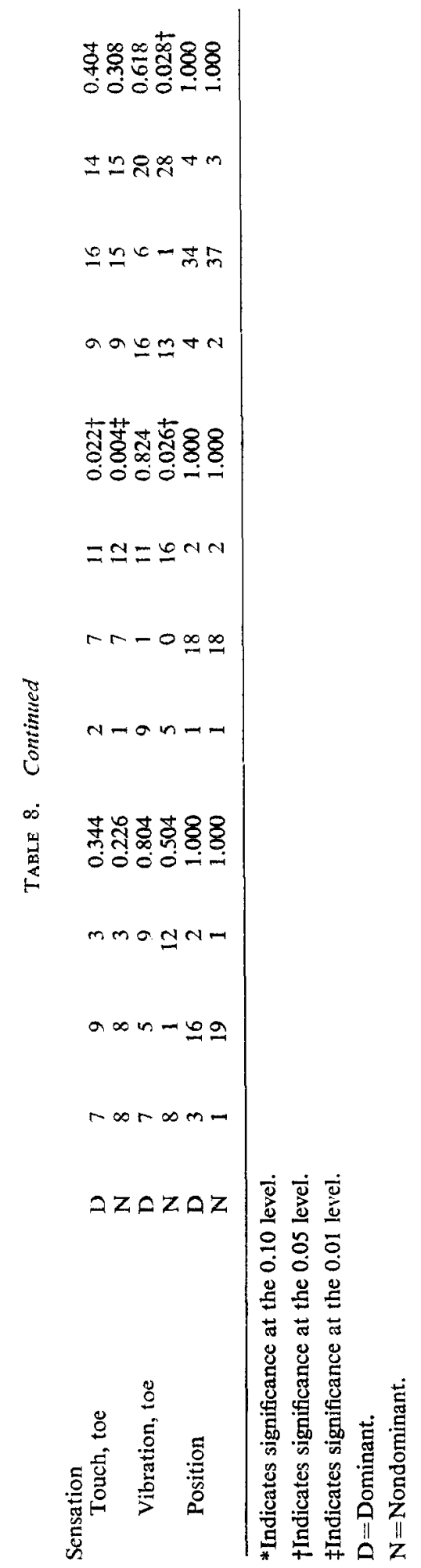


function ( $\frac{\text { patient score }}{\text { normative score }} \times 100$, with scores expressed in terms of tasks accomplished per second except for vocalization and drinking when the inverse ratio was used). See Table 6. It is then possible to determine how effective amantadine is in bringing the patient back to normal function. The average percentage of normal function for all 19 of the SADLE tests was 31.9 per cent pretreatment, 30.8 per cent after placebo treatment and 39.6 per cent after amantadine treatment.

\section{Clinical quantitative neurological examination (CQNE)}

A summary of the CQNE results is shown in Table 7. Control scores obtained from 80 asymptomatic young adults (mean age $=22.4 \mathrm{yr}$ ) are compared to patient scores upon entry (previous medication), pretreatment (no or minimal medication), and after placebo and amantadine treatment. Examination of the paired $t$-test analyses indicates a significant preference for amantadine in the following test areas:

\section{Strength of movements}

1. Grip strength, dominant and nondominant.

2. Shoulder abduction, dominant.

\section{Coordination of movements}

1. Speed-coordination of hand, dominant and nondominant.

2. Index of performance, dominant and nondominant hand.

3. Rotary pursuit tracking, dominant and nondominant hand.

4. Finger dexterity, Purdue pegboard and pencil rotation, dominant and nondominant.

5. Speed-coordination of foot, forward and side step, dominant and nondominant.

6. Index of performance, dominant and nondominant foot, forward and side step.

\section{Station and gait}

1. Standing, eyes open, two legs together.

2. Standing, eyes closed, two legs together.

3. Tandem gait, walking without and with support.

Results indicate specific improvement in all of the coordination tests, with improvement in some of the strength, station and gait tests. Significant changes were not observed for those tests measuring sensation (vision, touch, vibration, position or 2-point discrimination), steadiness, simple reaction time, speed of hand, strength of the lower extremities, or fatigue with the exception of increased fatigue as measured by one of the foot coordination fatigue tests. Comparison to the remainder of the fatigue tests suggests that this isolated finding is of little significance.

The results of a sign test analysis upon the CQNE scores after placebo and amantadine treatment are summarized in Table 8 . As before, any measurable improvement or deterioration was regarded as a change for the purposes of this analysis. Findings are similar to those using the $t$-test, indicating improved performance following amantadine treatment in some of the strength tests, the coordination tests, and unsupported tandem gait test, and two of the fatigue measures. A statistically significant difference also was found for amantadine treated patients for one of the vibration tests. This was the only significant sensory finding.

These results obtained from the SADLE and CQNE test batteries confirm the findings obtained with the subjective and qualitative methods (patients' impressions, 
neurologists' overall impression, functional disability scores, and evaluation of relevant physical signs). There is no question that amantadine is a better treatment than placebo for Parkinson's disease.

In order to simplify the analysis of the CQNE data and to express it in more clinical terms, functionally related tests were grouped into the following categories:

1. Vision (visual acuity).

2. Strength (grip, wrist dorsiflexion, shoulder abduction, fool dorsiflexion, hip flexion).

3. Steadiness (hole steadiness, supported and unsupported).

4. Reaction time (simple reaction time).

5. Speed (speed of hand, speed of foot).

6. Coordination (speed coordination of hand and foot; index of performance, hand and foot; rotary pursuit; Purdue pegboard; pencil rotation).

7. Fatigue (grip strength fatigue, hip strength fatigue, speed of hand and foot fatigue, speed-coordination of hand and foot fatigue).

8. Station (standing: eyes open, two legs and one leg; eyes closed, two legs and one leg).

9. Gait (tandem gait, supported and unsupported).

10. Sensation (touch, hand and toe; vibration, finger and toe; position, upper and lower extremities; 2-point discrimination).

The overall CQNE findings can be summarized by first expressing all of the test scores as a percentage of normal function $\left(\frac{\text { patient score }}{\text { normative score }} \times 100\right.$ or $\frac{\text { normative score }}{\text { patient score }} \times 100$ when better performance reflects a lower score, e.g., vibration sense), and then averaging all of the test scores representative of a functional category. For example, after expressing all of the strength test scores for a given patient in terms of 'percentage of normal function', the measure of grip strength, wrist dorsiflexion, shoulder abduction, etc., can be averaged to obtain a single measure of STRENGTH. The actual measures, expressed as percentage of normal function, are shown in Table 9. A summary of the change in percentage of normal function, comparing placebo and amantadine scores to pretreatment scores for the functional categories is shown in Fig. 4. The functional category demonstrating the most significant improvement is coordination, with strength and gait also demonstrating improvement, significant at $p<0.05$. None of the other functional categories show significant change.

\section{Neuro-psychological test battery (NPE)}

Of the 14 NPE tests administered, only one, the digit-symbol substitution test, demonstrated a significant difference between placebo and amantadine treatment. The digit-symbol test requires the subject to substitute a symbol for a digit, writing the symbol on a piece of paper. As such, the test does have significant motor output and is more than a measure of purely cognitive function. Moreover, of all the subtests in the Wechsler Adult Intelligence Scale, digit-symbol is most sensitive to changes in fine manual dexterity and visual-motor coordination. Performance following amantadine treatment was significantly better than after placebo treatment at the $p<0.05$ level. A comparison after placebo and amantadine treatment of those tests having quantitative measures is shown in Fig. 5. 


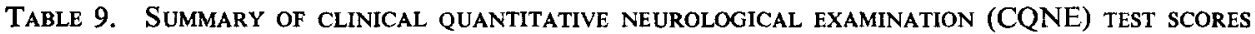
FOR THE COMBINED GROUPS SHOWN IN TABLE 7 EXPRESSED AS PERCENTAGE OF NORMAL FUNCTION ${ }^{1}$. PRETREATMENT, PLACEBO AND AMANTADINE SCORES

\begin{tabular}{lcccc}
\hline \multicolumn{1}{c}{$\begin{array}{c}\text { Functional } \\
\text { categories }\end{array}$} & Pretreatment & Placebo & Amantadine & $\begin{array}{c}\text { Paired } t \text {-test } \\
\text { Difference between } \\
\text { amantadine and placebo }\end{array}$ \\
\hline Vision & 91.0 & 87.4 & 88.1 & 0.70 \\
Strength & 60.0 & 62.6 & 64.4 & $2.69 \dagger$ \\
Steadiness & 51.1 & 54.9 & 53.6 & -0.55 \\
Reaction time & 62.2 & 66.4 & 67.3 & 0.89 \\
Speed & 58.3 & 60.6 & 62.4 & 1.20 \\
Coordination & 40.9 & 42.0 & 47.9 & $5.09 \ddagger$ \\
Fatigue & 91.7 & 93.7 & 94.3 & 0.40 \\
Station & 44.2 & 48.3 & 49.2 & 0.48 \\
Gait & 41.1 & 40.1 & 44.6 & $2.22 \dagger$ \\
Sensation & 75.8 & 75.3 & 76.6 & 0.66 \\
Total upper extremity & 65.1 & 66.9 & 68.5 & $2.24 \dagger$ \\
Total lower extremity & 59.1 & 61.4 & 63.3 & 1.44 \\
Combined upper and & & & & $2.02 \dagger$ \\
lower extremity & 62.1 & 64.2 & 65.9 & \\
\hline
\end{tabular}

fIndicates significance at the 0.05 level.

¥Indicates significance at the 0.01 level.

'As established by test scores obtained from 80 young adults.

\section{Factors infuencing response to amantadine}

The 27 patients who responded favorably to amantadine treatment were compared to the 15 patients who did not. The age ( $66 \mathrm{yr}$ vs. $64 \mathrm{yr}$ ), sex (63 per cent males in group responding vs. 62 per cent males in group not responding), and treatment group assignment ( 54 per cent of patients responding to amantadine in Group 1) were similar for both groups. The duration of disease was greater for those patients responding to amantadine than those who did not $(10 \mathrm{yr} v s .5 \mathrm{yr} ; p<0.05)$. In addition, the degree of disability as determined by the neurologists was greater in those patients responding to amantadine than those who did not, although this difference was not significant. The average stage of the group responding to amantadine was 3.1 while the average stage of the group that did not respond was 2.5. (See Hoehn et al [12] for the stages of Parkinsonism based on the level of clinical disability.) Subjective comparison of the six patients using concurrent anti-Parkinsonian medications suggests greater improvement for this group of subjects as compared to those patients using no concurrent medication. The small sample size (six patients) makes statistical analysis inappropriate. Evaluation of the General Disability Scale for these six patients indicates an improvement of 24 per cent after amantadine treatment when compared to pretreatment scores contrasted to a 9 per cent improvement for the remaining patients. However, analyses of quantitative data indicate no significant changes in the results when these six patients are not included in the evaluation.

\section{Side effects}

Treatment was not associated with important adverse side effects in any of the cases. As noted by other investigators [13], more side effects were reported with 


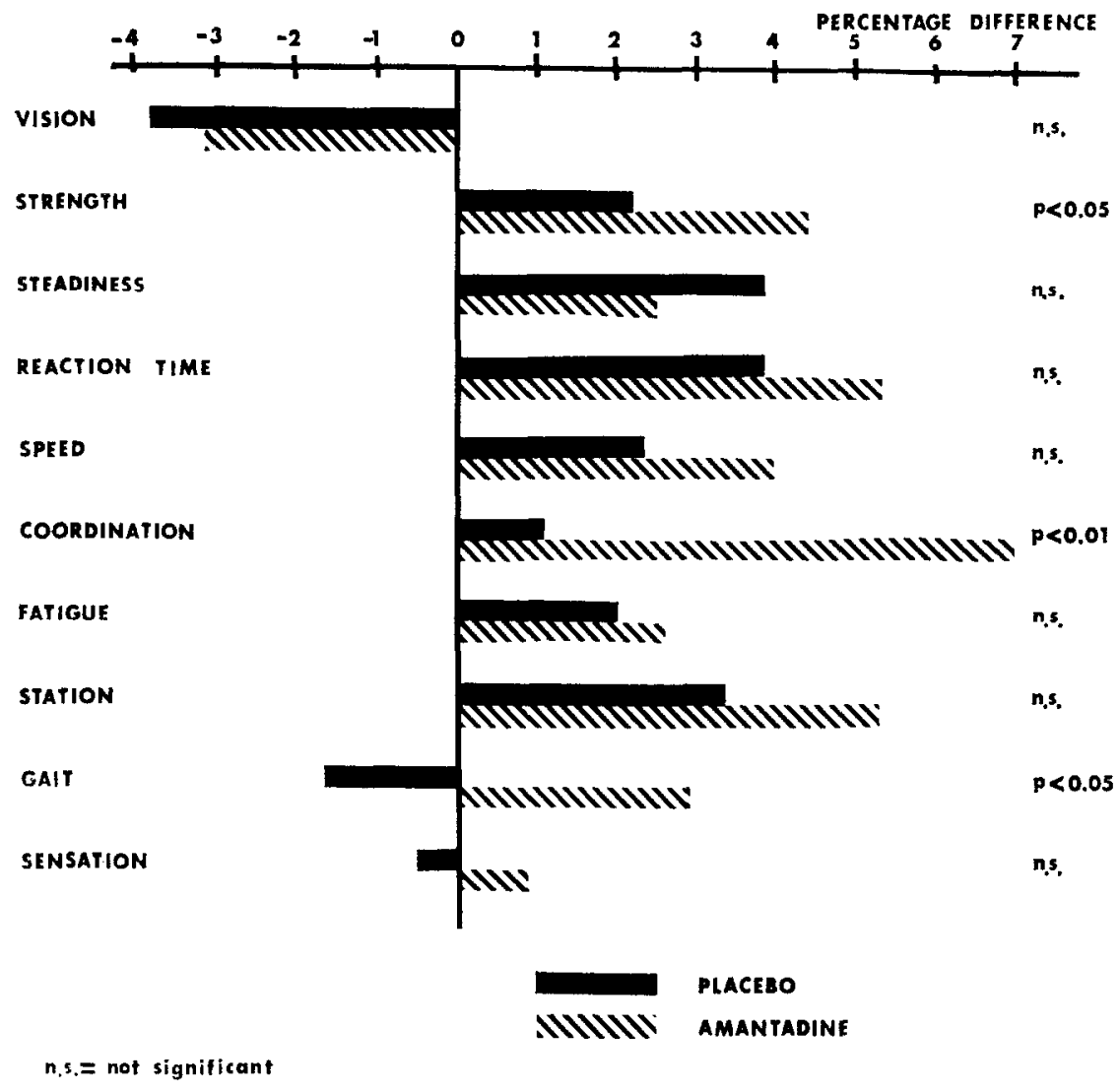

FIG. 4. Clinical quantitative neurological examination (CQNE) (functional categories). Comparison of percentage of normal function of placebo and amantadine treated patients when compared to pretreatment scores.

placebo treatment than with amantadine treatment. Results are shown in Table 10. Eight patients had a transient increase of leukocytes in their urine while on amantadine, although no patient had more than 15 cells per high power field. There were no symptoms of urinary tract infection.

\section{DISCUSSION}

The qualitative and quantitative measures employed in this trial demonstrate that amantadine is superior to placebo in the treatment of Parkinson's disease. Moreover, the benefit appears to be greater than that afforded by the patients' standard medications used upon entry to this study.

Walking, as evaluated by both patients and neurologists, improved more than the other subjective measures, but hygiene, eating, feeding and speech also improved. Clinical neurological evaluation demonstrated improvement in tremor, weakness, succession movements, rising, posture and gait. This broad improvement contrasts with the findings of Hunter et al [7] who reported only a small beneficial effect on physical signs and no significant effect upon functional abilities. The larger size of our series (42 vs. 12) may account for this disagreement. 


\section{PEALOPY PICTURE VocakUiaRY \\ RAVEN COLOURED MATRICES \\ HOPFER VISUAL ORGANIZATION \\ SYMEOL - DIGIT WRITTEN \\ SYMPOL-DIGIT \\ DIGIT SPAN \\ DIGIT - SYMBOL \\ pictuge
COMpletion \\ PICTURE \\ ARRANGEMENT \\ OAfET \\ ASSEMBLY}

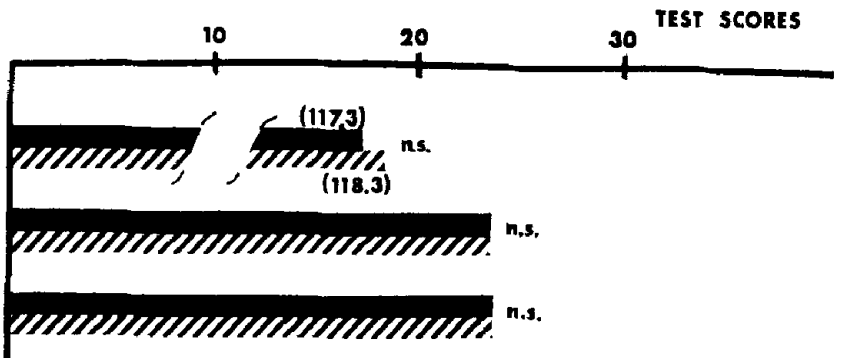

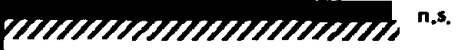

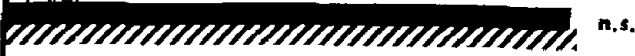

TIIINININ), n.s.

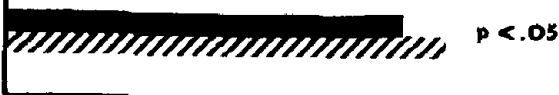

"renerener/, n.s.

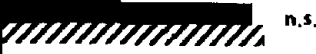

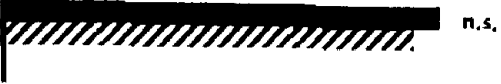

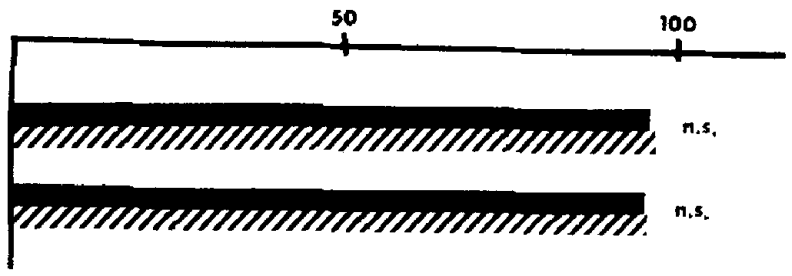

n.s.= not significant

PLACEBO

III/I/I. AMANFADINE

FIG. 5. Neuro-psychological examination (NPE), Effect of placebo and amantadine treatment on Parkinsonism.

Quantitative analysis of a broad range of motor, sensory, and cognitive tests revealed significant improvement only in the motor sphere. The most marked improvement was noted in tasks associated with fine finger manipulations, gross arm and leg movements, station, and gait. Overall strength also improved slightly, but significantly. The improvement in these basic abilities corresponded with improvement in the tests of simulated activities of daily living, a battery of tests designed to mimic tasks which patients must carry out in order to care for themselves. The agreement with subjective assessment establishes the consensual validity of the quantitative measures. Note that the specific areas of improvement could not have been determined without a comprehensive battery of motor, sensory, and psychological tests. 
TAble 10. Number of patients reporting side efFects During AMANTADINE AND PLACEBO TREATMENT

\begin{tabular}{lcc}
\hline & Placebo & Amantadine \\
\hline Nervousness & 2 & 0 \\
Anorexia & 0 & 1 \\
Weight loss & 2 & 4 \\
Insomnia & 4 & 0 \\
Easy fatigability & 3 & 1 \\
Dizziness & 4 & 1 \\
Headache & 1 & 0 \\
Loss of consciousness & 0 & 0 \\
Nausea, vomiting & 0 & 0 \\
Indigestion & 0 & 0 \\
Diarrhea & 0 & 0 \\
Constipation & 1 & 3 \\
Unsteadiness & 3 & 2 \\
Confusion & 1 & 1 \\
Depression & 1 & 1 \\
Early waking & 0 & 0 \\
Difficulty concentrating & 0 & 0 \\
Psychosis, hallucinations & 0 & 0 \\
Abnormal movements & 1 & 0 \\
Rash & 0 & 0 \\
Dry mouth & 2 & 0 \\
Blurred vision & 2 & 2 \\
Urinary straining & 2 & 2 \\
Edema & 1 & 0 \\
No side effects & 18 & 26 \\
\hline
\end{tabular}

Another important finding resulting from the use of a battery of tests is the security of knowing that the drug does not produce a decrement in performance in those areas not specifically affected. Talland [14], for example, found a diminution in certain cognitive functions (verbal rate, learning, Necker cube oscillation) in Parkinsonian patients using Artane ${ }^{\mathrm{R}}$ or Parsidol ${ }^{\mathrm{R}}$ compared with patients not using these drugs. We think a battery such as ours would be useful in evaluating any drug for the detection of deleterious nervous system side effects.

We emphasize that all mention of improvement has been expressed in terms of statistical significance. As is often the problem in any study involving a relatively large number of patients, statistically significant findings may result from small changes, so small as to be of little biological significance. Alternatively, in studying basic abilities such as strength, reaction time, or hand coordination, it is difficult to determine what effect small changes in these items will have upon the overall functional capacity of the patient. One major advantage of the quantitative measures expressed in interval or ratio units when contrasted with the subjective scales expressed in ordinal units involves the capability of expressing the patients' performance as a percentage of normal function. This permits the physician to establish more meaningful estimates involving the actual degree of improvement that can be expected following a specific drug trial. In this study, many of the measures evaluated in this fashion improved only slightly. However, the average increase (from 31.9 to 39.6 per cent of normal function) following amantadine treatment for the tests of simulated activities of daily living (Table 6) is probably of considerable biological significance to the individual patient. 
Another way of establishing drug efficacy is to compare a new drug with standard medications. In every category tested, amantadine performed as well as or better than the patients' standard, optimal drug therapy, though few of the comparisons reached statistical significance.

\section{SUMMARY}

A double-blind crossover trial of amantadine vs. placebo was carried out involving 42 patients with Parkinson's disease: 64 per cent of the patients on amantadine experienced subjective improvement compared to 21 per cent on placebo. A comprehensive battery of qualitative and quantitative tests was carried out on each patient on entry to the study, after previous standard treatment was discontinued or reduced to a minimal tolerable dose, while on placebo, and while on amantadine, at 3 week intervals. Almost all relevant symptoms and physical signs improved, and the neurologists judged amantadine superior to placebo in 74 per cent of the patients. Quantitative measurement revealed significant improvement in 10 of 19 tests of simulated activities of daily living, in several tests of strength and station, and in all tests of coordination and gait. When the amantadine scores were compared to the placebo scores, an average improvement of 29 per cent occurred in the simulated activities of daily living, 14 per cent in tests of coordination, 11 per cent for gait and 3 per cent for strength. Sensation and neuropsychologic performance were unaffected and side effects were minimal. Comparison of amantadine scores with entry scores obtained when the patients were on standard anti-Parkinsonian medications suggested that amantadine may also be superior to classical medications. The response to amantadine was not related to age, sex, or severity of disease, but those who responded were found to have a significantly longer duration of illness. Amantadine is a nontoxic, easily administered drug useful in the treatment of Parkinson's disease.

It should be emphasized that the quantitative tests used in this study yielded interval data. This resulted in more valid comparisons with normal, particularly when expressed in terms of the percent of the age-matched normal function.

Finally, this is the first report which describes a battery of quantitative tests designed to measure in part the effect of a drug on activities of daily living. It could be that these results were the most indicative of a significant effect in this experiment, since it is an improvement in the accomplishment of activities of daily living, not neurological tests, by which a patient with Parkinsonism bases his judgement of the effectiveness of a non-toxic treatment.

Acknowledgements - The authors wish to express appreciation to all who participated in this study. The assistance of Mrs. C. B. Kazmierczak, R.P.T. and Mrs. S. L. Shimp who participated in the CQNE administration and data collection is greatly appreciated as is the assistance of Miss $\mathrm{H}$. $\mathrm{B}$. Smith, O.T.R. who helped develop the SADLE test battery and Miss J. A. Sagath, O.T.R. who helped develop and administered the SADLE examination. In addition to assisting in test administration, Mr. R. Champoux, M.A. compiled and organized the neuropsychological data. We thank Mrs. S. R. Henderson, M.P.H. and Mrs. Dot N. Snyder for her assistance in the analysis phase of this study, and Mr. R. S. Stribley, M.S. for his technical assistance in designing and building much of the instrumentation used in the Clinical Quantitative Neurological Examination. In addition we thank Drs. K. R. Magee and T. A. Newkirk, University of Michigan, for their assistance and cooperation in allowing us to study their patients. We are grateful to Dr. S. A. Mahler of the E.I. du Pont de Nemours \& Company for the supply of amantadine and placebo and for her helpful discussions and support. 


\section{REFERENCES}

1. Finklea JF, Hennessy AV, Davenport FM: A field trial of amantadine prophylaxis in naturally acute occurring respiratory illness. Am J Epidem 85: 403-412, 1967

2. Wendel HA, Snyder MT, Pell S: Trial of amantadine in epidemic influenza. Clin Pharmacol Ther 7: 38-43, 1966

3. Schwab RS, England EC Jr, Poskanzer DC et al: Amantadine in the treatment of Parkinson's disease. JAMA 208: 1168-1170, 1969

4. Weeth JB, Shealy CN, Mercier DA: I-DOPA and amantadine in the therapy of Parkinsonism. Wisconsin Med J 68: 325-328, 1969

5. Parkes JD, Zilkha KJ, Marsden P et al: Amantadine dosage in treatment of Parkinson's disease, Lancet 1: 1130-1133, 1970

6. Fieschi $C$, Nardini $M$, Casacchia $M$ et al: Amantadine vs. 1-DOPA and amantadine plus l-DOPA. Lancet 2: 154-155, 1970

7. Hunter KR, Stern GM, Lawrence DR et al: Amantadine in Parkinsonism. Lancet 1:1127-1129, 1970

8. Canter CJ, De La Torre R, Mier M: A method for evaluating disability in patients with Parkinson's disease. J Nerv Ment Dis 133: 143-147, 1961

9. Henderson WG: Application of some statistical methods including factor analysis to a battery of clinical quantitative neurological tests for evaluating disability in multiple sclerosis. Ph.D. Thesis, University of Michigan, 1970

10. Rose AS, Kuzma JW, Kurtzke JF et al: Cooperative study in the evaluation of therapy in multiple sclerosis: ACTH vs. placebo in acute exacerbations. Preliminary report. Neurology (Minneap) 18: 1-20, 1968

11. Tourtellotte WW, Haerer AF, Simpson JF et al: Quantitative clinical neurological testing I. A study of a battery of tests designed to evaluate in part the neurological function of patients with multiple sclerosis and its use in a therapeutic trial. Ann NY Acad Sci 122: 480-505, 1965

12. Hoehn MM, Yahr MD: Parkinsonism: Onset, progression and mortality. Neurology (Minneap) 17: 427-442, 1967

13. Parkes JD, Zilkha KJ, Calver DM et al: Controlled trial of amantadine hydrochloride in Parkinson's disease. Lancet 1: 259-262, 1970

14. Talland GA: Cognitive function in Parkinson's disease. J Nerv Ment Dis 135: 196-205, 1962

\section{APPENDIX A \\ GENERAL DISABILITY SCALES}

Neurologists' Evaluation by Inquiry of Activities of Daily Living [8].

Always walks alone

Scale A. WALKING

Normal

Gait only slightly deviant from normal in quality and speed; turning is the most difficult task, posture essentially normal

Quality of gait is poor and rate is slow; posture moderately affected; there may be a tendency toward mild propulsion; turning is difficult

Gait is extremely abnormal; very slow and posture grossly affected; there may be propulsion

Sometimes walks alone

Walks short distances with ease; walking outdoors is difficult but often accomplished without help; rarely walks longer distances alone

Walks from room to room with only moderate difficulty; may occasionally walk outdoors without assistance

Walks from room to room without assistance, but moves slowly and uses external support; never walks alone outdoors

Never walks alone

Requires potential help indoors and active help outdoors

Requires moderate help indoors; walks outdoors with considerable help

Needs considerable help even for short distances; cannot walk outdoors with help

Cannot walk at all, even with maximum assistance 
Complete self help

Scale B. Dressing

Normal

Dress self completely with only slightly more time and effort than normal

Dress self completely with slowness and great effort

1

2

Requires partial assistance

Handles all dressing alone with the exception of fine activities (tie, buttons)

Performs more than half of dressing activities alone, with considerable effort and slowness

Performs about half of dressing activities independently

Performs only gross dressing activities alone (hat, coat)

Requires complete assistance

Gives considerable help through bodily movements

Can give some help through bodily movements

Movements of patient neither help nor hinder assistant

Patient is a hindrance rather than a help to assistant

Complete self help

Scale C. Hygiene

Normal

Hygiene maintained normally, with exception of slight slowness

Hygiene activities are moderately time-consuming; no substitute methods; few accidents

Hygiene maintained independently, but with effort and slowness; accidents are not infrequent, may employ substitute methods

Requires partial assistance

Manages most of personal needs alone; has substituted methods for accomplishing difficult tasks (electric razor)

Requires assistance for some tasks not difficult in terms of coordination

Requires assistance for half of toilet needs

Performs a few tasks alone with assistant nearby

Requires complete assistance

Hygiene maintained well; gives aid to assistant

Reasonably good hygiene with assistance, but does not provide assistant with significant help

Unable to maintain proper hygiene with even maximum help

Scale D. EATING AND FEEDING

Eating

Normal

Follows a normal diet, but chewing and swallowing are labored

Eats some hard foods routinely, but these require time and effort

Liquids and soft foods handled with ease, hard foods occasionally eaten, but requirc great effort and much time

Eats only liquids and soft foods; these are consumed very slowly

Eating so impaired that a hospital setting is required to get adequate nutrition

\section{Feeding}

Normal

Fully feed self with rare accidents, slower than normal

Handles all feeding alone with moderate slowness; still may get assistance in specific situation (cutting meat in restaurant); accidents are not infrequent

Performs most feeding activities alone, slowly and with effort; requires help with specific tasks (cutting meat, filling cup)

Performs only a few tasks independently

Requires complete assistance 
Speech

Scale E. SPEECH

Normal

0

Speech entirely adequate; minor voice disturbances present

Speech easily understood, but voice or speech rhythm may be disturbed

Communication accomplished with ease, although speech impairment detracts from content

Speech can always be understood if listener pays close attention; both articulation and voice may be defective

Speech always employed for communication, but articulation is still very poor; usually uses complete sentences

Uses speech for most communication, but articulation is highly unintelligible; may have occasional difficulty in initiating speech; usually speaks in single words or short phrases

Attempts to use speech for communication, but has difficulty in initiating vocalization; may stop speaking in middle of phrase and be unable to continue

Vocalizes to call attention to self

Vocalizes but rarely for communicative purposes

Does not vocalize at all

\section{APPENDIX B}

Simulated activities of DaILY living EXAMINATION (SADLE)* aNd abBreviations $\dagger$

\begin{tabular}{rllr}
\hline \multicolumn{1}{c}{ Test } & Abbreviation & $\begin{array}{r}\text { Time limit } \\
(\mathrm{sec})\end{array}$ \\
\hline 1. Putting on a shirt & Shirt & 300 \\
2. Managing visible buttons; 1 in button & Button (large) & 120 \\
3. Managing visible buttons; $\frac{1}{2}$ in button & Button (small) & 120 \\
4. Opening and closing a zipper & Zipper & 60 \\
5. Tying a bow & Bow & 120 \\
6. Cutting with a knife & Cutting & 120 \\
7. Using a fork & Fork & 60 \\
8. Pouring water into a glass & Pouring & 120 \\
9. Squeezing toothpaste from a tube & Toothpaste & 60 \\
10. Dialing a telephone & Dialing & 60 \\
11. Opening and closing a safety pin & Safety pin & 120 \\
12. Opening and closing a door & Door & 60 \\
13. Opening an envelope & Envelope & 60 \\
14. Drinking from a glass with a straw & Drinking & 60 \\
15. Prolonged vocalization & Vocalizing & -120 \\
16. Washing hands; using dominant hand & Scrub-D & 60 \\
17. Putting on gloves; using dominant hand & Gloving-D & \\
& $\quad$ using nondominant hand & Gloving-N & \\
\end{tabular}

*Scores are obtained by averaging two timed trials for all tests with the exception of the toothpaste and drinking tests where one timed trial is used.

†For a complete description of SADLE tests see: Potvin, A. R.: The Effects of Age, Motivation and Learning on Performance in the Quantitative Examination of Neurological Function. Ph.D. Thesis, University of Michigan, 1971. 


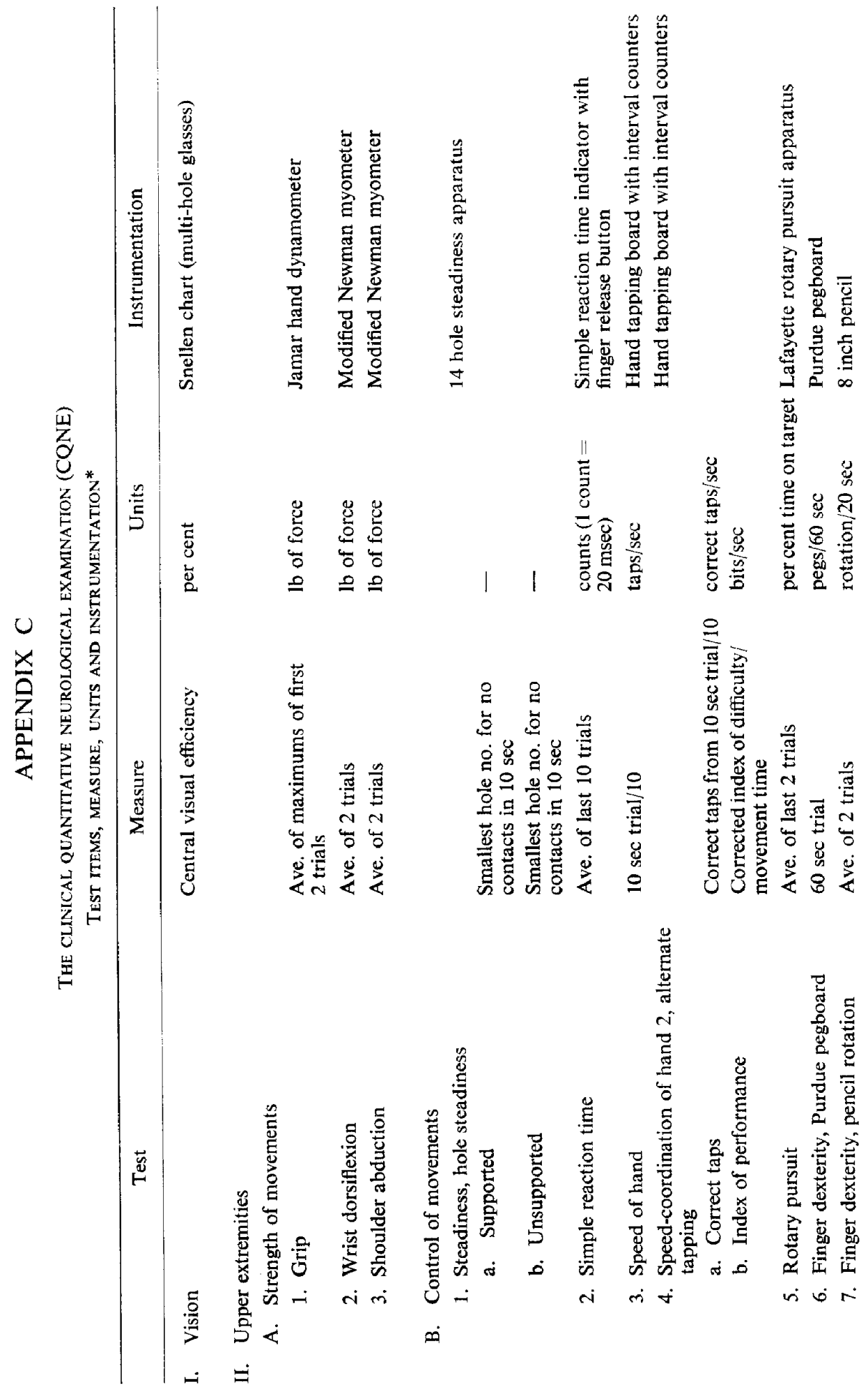



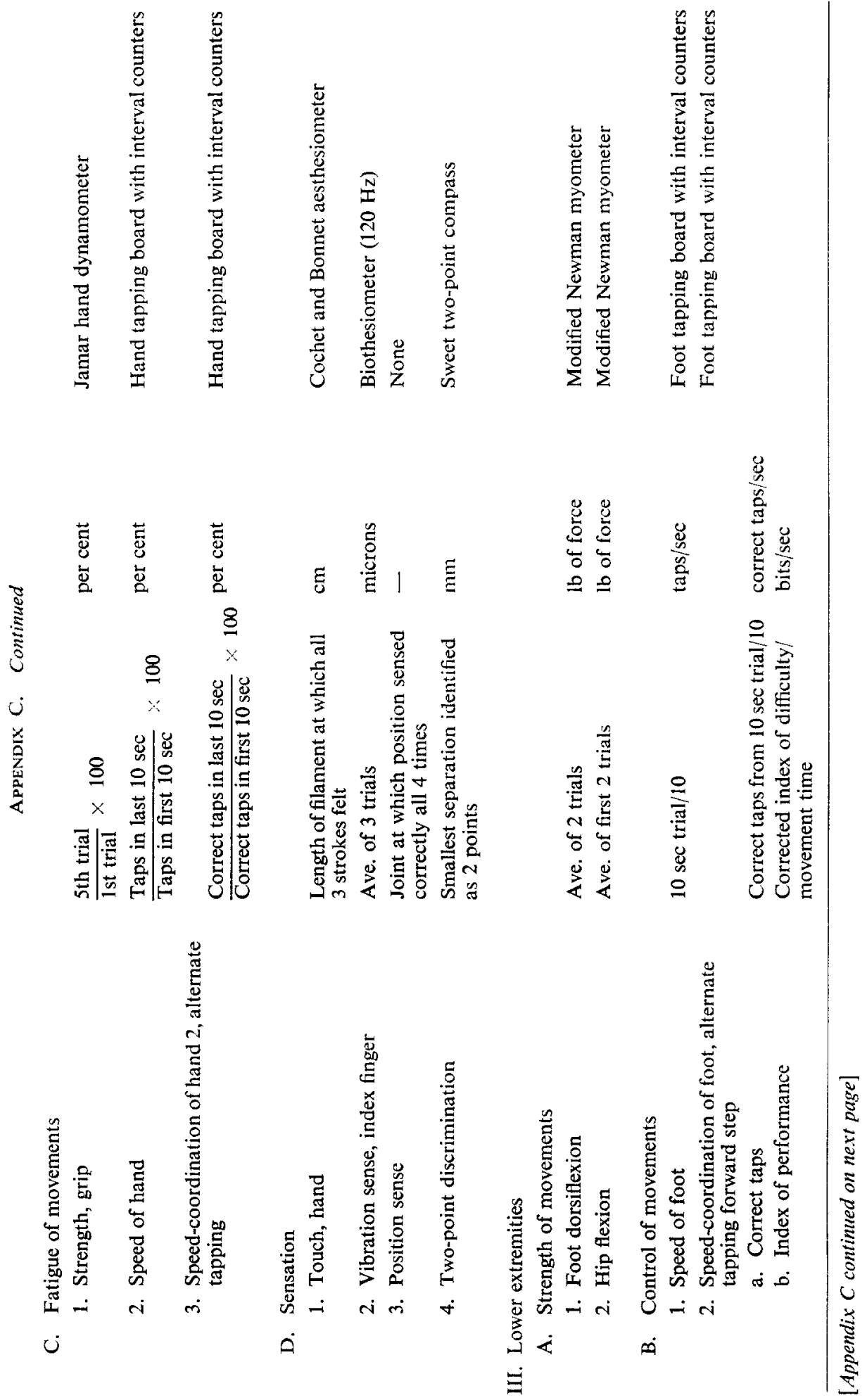


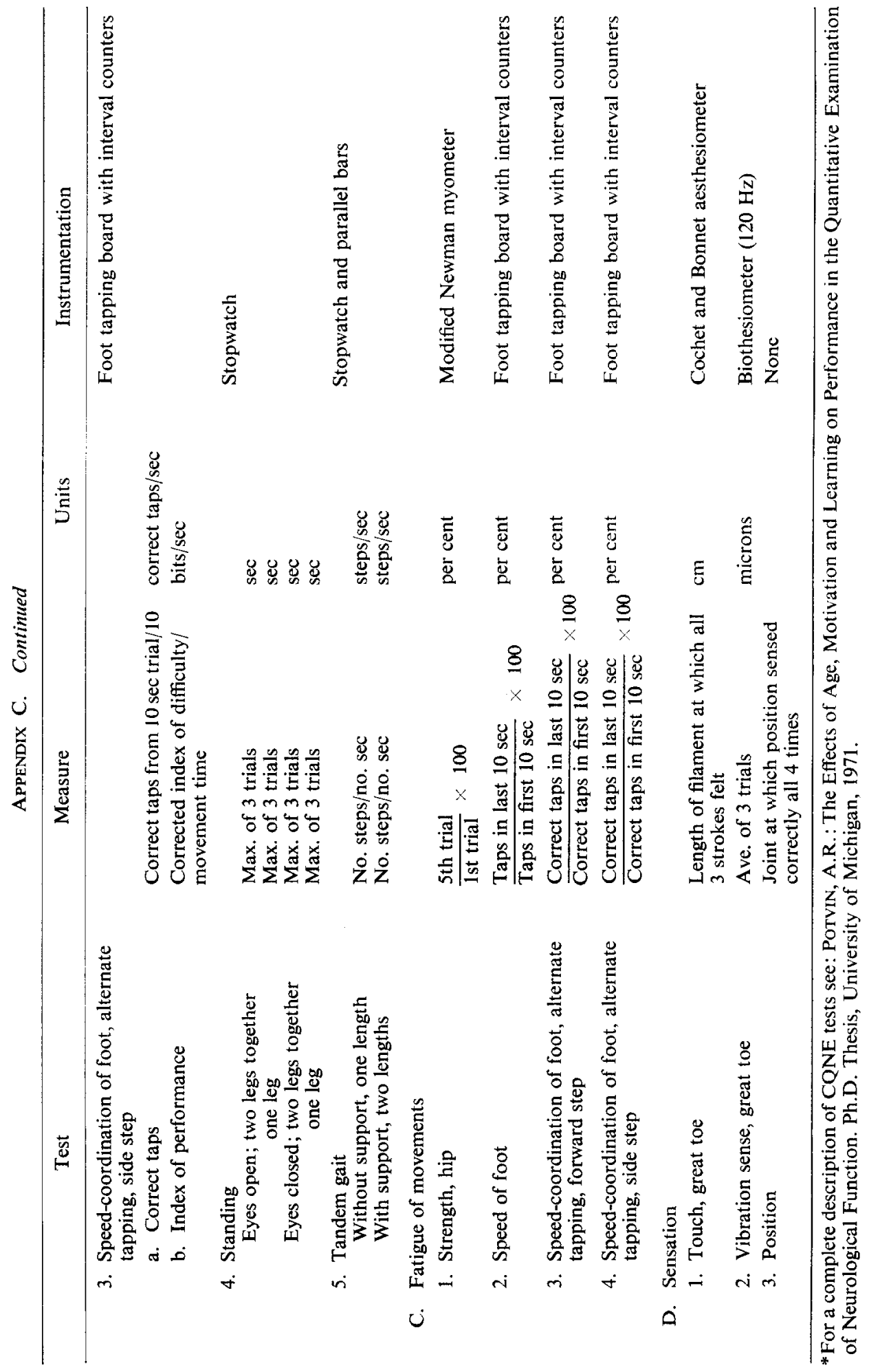

